\title{
The Dynamic Black-Litterman Approach to Asset Allocation
}

\author{
Richard D.F. Harris \\ University of Exeter \\ Evarist Stoja \\ University of Bristol \\ Linzhi Tan \\ Nottingham Trent University
}

October 2016

\begin{abstract}
We generalise the Black-Litterman (BL) portfolio management framework to incorporate time-variation in the conditional distribution of returns in the asset allocation process. We evaluate the performance of the dynamic BL model using both standard performance ratios as well as other measures that are designed to capture tail risk in the presence of non-normally distributed asset returns. We find that the dynamic BL model outperforms a range of different benchmarks. Moreover, we show that the choice of volatility model has a considerable impact on the performance of the dynamic BL model.
\end{abstract}

JEL Classification: C22, C53, G11

Keywords: Black-Litterman model; Multivariate conditional volatility; Portfolio optimization; Non-normality; Tail risk.

Address for correspondence: Professor Richard D. F. Harris, Xfi Centre for Finance and Investment, University of Exeter, Exeter EX4 4ST, UK. Tel: +44 (0) 1392263215, Email: R.D.F.Harris@exeter.ac.uk. Linzhi Tan, Division of Accounting \& Finance, Nottingham Business School, Nottingham Trent University, Burton Street, NG1 4BU, UK. Email: linzhi.tan @ntu.ac.uk. Evarist Stoja, School of Economics, Finance and Management, University of Bristol, 8 Woodland Road, Bristol, BS8 1TN, UK. Email: E.Stoja@bristol.ac.uk. We would like to thank Emanuele Borgonovo (the editor), two anonymous referees and seminar and conference participants at the University of Bristol, Nottingham Trent University and the 2016 Financial Econometrics and Empirical Asset Pricing Conference at Lancaster University for helpful comments and suggestions. Parts of this paper were written while Evarist Stoja was a Houblon-Norman Fellow at the Bank of England whose hospitality is gratefully acknowledged. The views expressed here are solely our own and do not necessarily reflect those of the Bank of England. 


\section{Introduction}

The portfolio theory proposed by Markowitz (1952) is a cornerstone of modern finance. Markowitz argues that investors should balance risk and expected return to determine the optimal allocation of assets. However, when implemented in practice, the resulting portfolio suffers from various problems, including extreme weights (Green and Hollifield, 1992), corner solutions leading to highly concentrated portfolios (Grauer and Shen, 2000), sensitivity of the solution to the input parameters and large fluctuations in the weights over time (Best and Grauer, 1991). The main reason for this is estimation error in the inputs of the model, which has a disproportionate effect on the resulting portfolio weights. Kolm et al. (2014) summarise several methods that have been widely adopted in the literature to mitigate the impact of estimation error. These include Bayesian approaches and the Black-Litterman model (Jorion, 1991; Black and Litterman, 1991), robust optimization methods (Tütüncü and Koenig, 2004; Stinstra and Hertog, 2008; Huang et al., 2010), incorporating higher moments and tail-risk measures (Harvey et al., 2010), and imposing constraints on the portfolio weights (Jagannathan and Ma, 2003). In this paper, we focus on the Black-Litterman (1991, 1992, hereafter BL) model, which uses an equilibrium approach to estimate the expected returns of individual assets, incorporating the investor's views by adjusting the equilibrium expected returns using a Bayesian approach. The BL model overcomes many of problems associated with the mean-variance model and, as a result, has become one of the most commonly used asset allocation approaches in practice (Bevan and Winklemann, 1998; Bertsimas et al, 2012). In particular, the BL model provides stable and intuitively appealing mean-variance efficient portfolios based on the investor's subjective views, and eliminates the input sensitivity of mean-variance optimization (see also Herold and Maurer, 2003).

The assumptions that are implicit in the BL model include: (1) the expected return vector and the covariance matrix are constant over time, (2) the returns of individual assets are normally distributed, and (3) investors do not differentiate between positive and negative deviations from the mean. In reality, all three of these assumptions are questionable. For example, Bollerslev et al. (1988) argue that investors have time-varying conditional expectations of returns. Moreover, it is well known that the variances and covariances of most financial time series are time-varying (Andersen et al., 2004). In addition, there is overwhelming empirical evidence that asset returns are not normally distributed (Peiro, 1999; Ang and Chen, 2002), which would suggest that the standard deviation may not be a suitable measure of risk. As a result, alternative risk measures such as value at risk (VaR) and conditional value at risk 
(CVaR) have been developed (Artzner et al., 1999; Rockafellar and Uryasev, 2002). Relatedly, when returns are not normally distributed, the Sharpe ratio is no longer an appropriate measure of portfolio performance. To overcome this problem, a number of other performance ratios have been proposed (see, among others, Farinelli and Tibiletti, 2008; Farinelli et al., 2008; Farinelli et al., 2009). For example, the standard deviation has been replaced by $\mathrm{VaR}$ and CVaR measures of tail risk (Biglova et al., 2004; Rachev et al. 2007; Giacometti et al., 2007). Finally, evidence suggests that investors have asymmetric attitudes towards upside and downside risks (Scott and Horvath, 1980). Mitton and Vorkink (2007) show that investors are willing to sacrifice portfolios with higher Sharpe ratios for those with higher skewness. Consequently, a number of researchers have incorporated skewness and kurtosis into portfolio selection (Harvey et al., 2010; Kerstens et al., 2011), while others have incorporated measures of tail-risk. For example, Goh et al. (2012) separate the distribution of asset returns into positive and negative half-spaces and define a new robust risk measure, called Partitioned Value-at-Risk (PVaR), that incorporates asymmetry in the return distribution. They then select the portfolio with the minimum-PVaR. Favre and Galeano (2002) introduce Modified VaR to measure tail risk for distributions with asymmetry and fat tails, and then construct the optimal portfolio, which has the maximal ratio of expected excess return to Modified VaR. Similarly, Rachev et al. (2003) propose a Generalised Sharpe ratio that uses Conditional VaR to measure tail risk, and maximise this ratio to yield the optimal portfolio.

A number of recent studies have attempted to extend the BL model to deal with nonnormality in the distribution of asset returns (Giacometti et al., 2007; Martellini and Ziemann, 2007; Meucci, 2009), and in investors' views (Fabozzi et al., 2006; Beach and Orlov, 2007; Palomba, 2008; Babameto and Harris, 2009; Chiarawongse et al., 2012). However, none of these studies allows for time-variation in the conditional distribution of returns. Moreover, despite allowing for non-normality in returns, they evaluate the performance of the BL model using measures that are only valid under normality, namely the standard deviation and the Sharpe ratio.

We make the following contributions. First, we relax the assumption of a constant expected return vector and covariance matrix in the BL model. In particular, we generalize the BL model to incorporate time-variation in the conditional distribution of returns in the asset allocation process. Second, we relax the assumption of normally distributed returns and explicitly account for tail risk in the BL model. Moreover, we use alternative performance 
ratios that are appropriate for tail risk, namely the reward-to-VaR and reward-to-CVaR ratios, in order to construct the optimal dynamic BL portfolio. Third, we extend the method of Giacometti et al. (2007) to a dynamic setting and use the estimated time-varying VaR and CVaR to substitute the time-varying standard deviation in the quadratic utility function. Fourth, we estimate the tail risk-adjusted equilibrium returns and combine these with the investor's views to construct the dynamic BL portfolio.

We evaluate the out-of-sample performance of the following dynamic BL portfolios: the implied BL portfolio with reverse optimization, the SR-BL portfolio with maximal Sharpe ratio, the MVaR-BL portfolio with maximal reward-to-VaR ratio, the MCVaR-BL portfolio with maximal reward-to-CVaR ratio and the risk-adjusted $\mathrm{BL}$ portfolio. The market portfolios are represented by the 10 FTSE sector indices for the US, UK and Japan. To estimate the time-varying distribution of returns, we use a range of conditional volatility models (the Rolling Window, Exponentially Weighted Moving Average and Dynamic Conditional Correlation (DCC) models), distributional assumptions (the normal and tdistributions) and confidence levels (90\%, 95\% and 99\%). We report three main findings. First, the dynamic BL portfolios and risk-adjusted BL portfolios outperform the benchmark and the equally-weighted naive portfolio. Second, the dynamic BL portfolio based on the DCC model has the best out-of-sample performance. Third, the portfolios that account for tail risk outperform the portfolios that ignore tail risk. Our results are robust to the choice of estimation window length and data frequency, and hold in sub-samples.

The outline of the remainder of the paper is as follows. Section 2 describes the data used in the empirical analysis. Section 3 describes the dynamic BL asset allocation framework. Section 4 reports and discusses the empirical results and summarizes our findings. Section 5 presents the robustness tests. Section 6 offers some concluding remarks.

\section{Data}

We use monthly price indices and market values for the 10 FTSE industry sectors in the US, UK and Japan obtained from DataStream, for the period from December 1993 to August 2015, i.e. 261 observations. The FTSE sector indices are free from survivorship bias by construction. The price indices and market values are measured in US Dollars. In addition, we obtained the US one-month Treasury Bill rate for the corresponding period from the Kenneth R. French Data Library. We use the price indices to compute simple returns and then subtract 
the Treasury Bill rate to give excess returns, which are used throughout the empirical analysis. We employ the market value of each FTSE sector index to compute the weight of each index in the benchmark portfolio during each month.

Table 1 reports the summary statistics for the excess returns for each index from January 1994 to August 2015. The average standard deviation of excess returns across all sectors is $6.8 \%$ for the UK, $5.7 \%$ for the US and $6.8 \%$ for Japan, and ranges from $4.1 \%$ for US Health Care to $11.8 \%$ for UK Oil and Gas. Excess returns are leptokurtic for all 30 country-sectors, most notably for UK Financials, UK Basic Materials and Japan Telecom. They are negatively skewed for the majority of sectors in the US and UK, but positively skewed for all but one of the sectors in Japan. The null hypothesis of normality is rejected in 24 out of 30 cases at the five percent significance level, and in 19 cases at the one percent level.

[Table 1]

Table 2 reports the time series properties of excess returns for each index from January 1994 to August 2015. In particular, it shows the first five autocorrelation coefficients and the value of the Ljung-Box test for serial correlation up to 10 lags, the ARCH test of Engle (1982) and the DCC test of Engle and Sheppard (2001). In only a few cases do excess returns display significant autocorrelations. This suggests that it is only for these series that we need to specify a conditional mean model to predict excess returns. However, taking the low power of the test and the possibility of non-linear dependence in excess returns into account, we use a momentum strategy for all 30 indices in order to capture predictability in excess returns as a basis for the investor's views in the BL model (see Fabozzi et al., 2006). The results of the ARCH test suggest that there is significant volatility clustering for most of the excess return series. As shown in Table 2, the DCC test for all 30 excess return series fails to reject the null of a constant correlation in favour of a dynamic structure, with a p-value larger than $10 \%$. We apply the DCC test to the 18 indices that exhibit a strong volatility clustering effect. The test suggests that these indices exhibit significant time-varying conditional correlations, with a pvalue less than $1 \%$, thus motivating the use of dynamic conditional covariance models. The non-rejection of the null hypothesis for the remaining 12 series may be due to the low power of the test. Overall, therefore, the results reported in Table 2 suggest that it is appropriate to use a conditional volatility model to estimate the covariance matrix in the dynamic asset allocation model. 
[Table 2]

\section{Theoretical Framework}

We generalize the BL model in a dynamic asset allocation framework, and measure tail risk under the assumption of both normally distributed and t-distributed returns. The dynamic BL portfolios that we consider include both unconstrained as well as risk-adjusted portfolios. To construct the unconstrained BL portfolios, we calculate the optimal weights using the following methods: reverse optimization implied in the BL model (the implied BL portfolio); optimization with a maximal Sharpe ratio (the SR-BL portfolio); optimization with a maximal reward-to-VaR ratio (the MVaR-BL portfolio); and optimization with a maximal reward-to-CVaR ratio (the MCVaR-BL portfolio).

\subsection{Dynamic BL Model}

In the generalized dynamic BL model that we propose, we define the first and second moments of the excess returns of $N$ assets, conditional on the information set $Y$, as follows:

$$
\begin{gathered}
\mathbf{r}_{t}=\boldsymbol{\mu}_{B L, t}+\boldsymbol{\varepsilon}_{t} \\
\boldsymbol{\varepsilon}_{t} \mid Y_{t-1} \sim G\left(0, \mathbf{V}_{t}\right) \\
\boldsymbol{\mu}_{B L, t} \sim G\left(\boldsymbol{\pi}_{t}, \tau \mathbf{H}_{t}\right)
\end{gathered}
$$

where $\mathbf{r}_{t}=\left(r_{1 t}, r_{2 t}, \cdots, r_{N t}\right)^{\prime}$ is the $N \times T$ excess returns vector, $\boldsymbol{\mu}_{B L, t}$ is the $N \times 1$ vector of expected excess returns in period $t, \boldsymbol{\varepsilon}_{t}$ is the $N \times 1$ error term vector, $Y_{t-1}$ is the information set in the period $t-1 . G($.$) is any location-scale family distribution, \mathbf{V}_{t}$ is the $N \times N$ covariance matrix, $\boldsymbol{\pi}_{t}$ is the $N \times 1$ conditional equilibrium return vector of the market portfolio, and the scale parameter $\tau$ indicates the uncertainty of the CAPM prior. The smaller the value of $\tau$, the higher the confidence in the estimation of the implied equilibrium return. In Black and Litterman (1992) $\tau$ ranges between 0.01 and 0.05 .

\subsubsection{Estimation of the Time-Varying Covariance Matrix}

In the dynamic asset allocation model, we use a time-varying conditional volatility model to estimate the covariance matrix. Indeed, Table 2 clearly shows that for the majority of the excess return series, the data exhibit volatility clustering and time-varying conditional 
correlations. The literature is replete with covariance matrix forecasting models. In order to narrow the scope of the research, we select three widely used models in increasing order of sophistication. The Rolling Window (RW) model is the simplest but it also suffers from a number of limitations such as ghost features (see Alexander, 1998). The Exponentially Weighted Moving Average (EWMA) model provides a more realistic approach to the weighting of past observations, while preserving the simplicity of the RW model. This model puts more weight on recent observations relative to those in the distant past and remedies the 'ghost features' problem of the Rolling Window model (see Alexander, 1998), and is better suited to capturing volatility persistence. Moreover, EWMA-based VaR forecasts have been shown to be superior to those based on GARCH models in many cases (see, for instance, Alexander and Leigh, 1997; Boudoukh et al., 1997; Guermat and Harris, 2002). Finally, to capture time-varying conditional correlations in asset returns, we also employ the more sophisticated Dynamic Conditional Correlation (DCC) model (see, for instance, Engle and Sheppard, 2001; Engle, 2002; Kalotychou et al., 2014). An advantage of this model that it has a smaller number of parameters compared to traditional multivariate models such as the VEC and BEKK models, and can therefore be applied to problems involving a large number of assets. We rebalance the portfolio every month using the estimated conditional covariance matrix for that month.

\section{Rolling Window Covariance Matrix}

The Rolling Window (RW) covariance matrix is given by:

$$
\mathbf{H}_{t}=\frac{1}{M-1} \sum_{j=1}^{M}\left(\mathbf{r}_{t-j}-\overline{\mathbf{r}}\right)\left(\mathbf{r}_{t-j}-\overline{\mathbf{r}}\right)^{\prime}
$$

where $\overline{\mathbf{r}}=\frac{1}{M} \sum_{j=1}^{M} \mathbf{r}_{t-j}$ is the $N \times 1$ vector of sample mean excess returns within the window $t-M, \ldots, t-1$.

\section{EWMA Covariance Matrix}

The EWMA covariance matrix is given by:

$$
\mathbf{H}_{t}=\lambda \mathbf{H}_{t-1}+(1-\lambda) \mathbf{r}_{t-1} \mathbf{r}_{t-1}{ }^{\prime}
$$


where $\lambda$ is the decay factor, with $0 \leq \lambda \leq 1$, which determines how rapidly the weights on past observations decline, and is typically estimated to be between 0.92 and 0.96 . In RiskMetrics (J.P. Morgan, 1994), the decay factor is set to 0.94 .

\section{Covariance Matrix}

The DCC covariance matrix is given by:

$$
\mathbf{H}_{t}=\mathbf{D}_{t} \mathbf{R}_{t} \mathbf{D}_{t}
$$

where $\mathbf{H}_{t}$ is the time-varying covariance matrix, $\mathbf{D}_{t}$ is the diagonal matrix of time-varying standard deviations from univariate GARCH models with $\sigma_{i, t}$ as the $i^{\text {th }}$ element at time $t$, i.e., $\mathbf{D}_{t}=\operatorname{diag}\left\{\sigma_{i, t}\right\}, \mathbf{R}_{t}$ is the time-varying correlation matrix (see, Engle, 2002).

\subsubsection{Conditional Equilibrium Returns}

Bollerslev et al. (1988) argue that investors have conditional expected returns that are timevarying. They employ a multivariate GARCH process within the CAPM framework to estimate expected returns. The idea is that the expected returns are proportional to the conditional non-diversifiable risk represented by the conditional covariance of each return with the market portfolio.

Following Bollerslev et al. (1988), let $\mathbf{r}_{t}$ be the $N \times 1$ vector of excess returns of all assets in the market at time $t$, let $\boldsymbol{\pi}_{t}$ be the $N \times 1$ conditional mean vector and let $\mathbf{H}_{t}$ be the $N \times N$ conditional covariance matrix of these returns given information available at time $t-1$. In addition, define $\mathbf{w}_{t-1}$ to be the $N \times 1$ vector of market capitalization weights at time $t-1$, and hence the excess return on the market portfolio is $r_{M, t}=\mathbf{w}_{t-1} \mathbf{r}_{t}$. When the CAPM holds, the conditional mean vector $\boldsymbol{\pi}_{t}$ satisfies the following equation:

$$
\boldsymbol{\pi}_{t}=\delta_{t} \mathbf{H}_{t} \mathbf{w}_{t-1}
$$

where $\delta_{t}$ is the dynamic risk aversion coefficient. Brandt and Wang (2003) argue that the risk aversion coefficient is time-varying. We use a simple method to calculate the risk 
aversion coefficient as the value of the global market risk premium divided by the market variance (see Idzorek, 2004; Babameto and Harris, 2009).

\subsubsection{The Investor's Views}

An investor can possess views about some or all of the returns of the assets in a portfolio that may differ from the implied equilibrium returns. The uncertainty of the views is given by the error vector $\mathbf{e}_{t}$ with a mean of zero and covariance matrix $\boldsymbol{\Omega}$. The error terms are unknown and independent. The investor's views at time $t$ can thus be expressed as:

$$
\mathbf{q}_{t}=\mathbf{P}_{t} \boldsymbol{\mu}_{B L, t}+\mathbf{e}_{t}
$$

At time $t$, let $K \leq N$ be the total number of the views (including both relative and absolute views), $\mathbf{P}_{t}$ is the $K \times N$ matrix of view portfolios and $\mathbf{q}_{t}$ is the $K \times 1$ vector of expected returns on the view portfolios.

Following Fabozzi et al. (2006), we utilize a momentum strategy to generate views. However, we extend their approach by substituting the constant standard deviation with a time-varying standard deviation to calculate the dynamic normalized returns. Further, there is evidence that the momentum effect is strongest at the six-month horizon (see Richard, 1997). Thus, we rank securities over the past six months and the momentum portfolios are formed at time $t$ and held for 6 months. Therefore, the normalized six-month return $Z_{i, t}$ is given by:

$$
Z_{i, t}=\frac{p_{i, t-1}-p_{i, t-6}}{p_{i, t-6} \sigma_{i, t}}
$$

where $p_{i, t-1}$ is the sector index price $i$ at time $t-1 ; p_{i, t-6}$ is the sector index price $i$ at time $t-6$; and $\sigma_{i, t}$ is the volatility of the sector index price $i$ at time $t$. The top half of the sector indices are allocated weights of $\omega_{i, t}=\frac{1}{\sigma_{i, t} c}$, while the bottom half of the sector indices are allocated weights of $\omega_{j, t}=-\frac{1}{\sigma_{j, t} c}$, where, $i, j=1, \ldots, 30, i \neq j$. Then, the view matrix $\mathbf{P}_{t}$ in the BL model is a $1 \times N$ vector with each element equal to one of these two quantities, and thus represents the relative view that the top half of the sector indices will continue to 
outperform the bottom half of the sector indices. The parameter $c$ is a constant whose role is to constrain the annual long-short portfolio volatility to a certain level (20\% in this case). Note that the portfolio weights do not sum to zero with this non-zero-cost long-short portfolio. The expected return of this momentum portfolio is the expected view return $\mathbf{q}$. After constructing the momentum portfolio in each period $t$, we hold it for one month and observe its return $r_{m, t}$ over the holding period. For the same holding period, we also observe the realized return $r_{a, t}$ on the portfolio of the actual winners and losers. Then, the residual return is calculated as the difference between $r_{m, t}$ and $r_{a, t}$. The residual return series $\mathbf{e}_{t}$ is then obtained by rolling the evaluation window forward one month and repeating the process. The level of confidence in the views $\boldsymbol{\Omega}$ is equal to the variance of the series of residuals $\mathbf{e}_{t}$.

\subsubsection{Combining Conditional Equilibrium Returns and Views}

The next step in the estimation is to combine the conditional equilibrium returns with the views using a Bayesian approach. In the dynamic case, the $N \times 1$ vector of conditional expected returns $\boldsymbol{\mu}_{B L, t}$ at time $t$ is given by:

$$
\boldsymbol{\mu}_{B L, t}=\boldsymbol{\pi}_{t}+\tau \mathbf{H}_{t} \mathbf{P}_{t}^{\prime}\left(\mathbf{P}_{t} \mathbf{H}_{t} \mathbf{P}_{t}^{\prime} \tau+\boldsymbol{\Omega}_{t}\right)^{-1}\left(\mathbf{q}_{t}-\mathbf{P}_{t} \boldsymbol{\pi}_{t}\right)
$$

and the estimated $N \times N$ covariance matrix $\mathbf{V}_{t}$ is given by:

$$
\mathbf{V}_{t}=\mathbf{H}_{t}+\left(\left(\boldsymbol{t} \mathbf{H}_{t}\right)^{-1}+\mathbf{P}_{t}^{\prime} \mathbf{\Omega}_{t}^{-1} \mathbf{P}_{t}\right)^{-1}
$$

\subsection{Unconstrained Dynamic BL Portfolio}

We estimate the time-varying expected returns and covariance matrix from Equations (8) and (9). We use two methods to construct the unconstrained BL portfolio at each period $t$. For the

first method, we use reverse optimization to compute the implied weights $\mathbf{w}_{B L, t}^{*}$ at time $t$, given by:

$$
\mathbf{w}_{B L, t}^{*}=\frac{1}{\delta_{t}} \mathbf{V}_{t}^{-1} \boldsymbol{\mu}_{B L, t}
$$


(see, for example, Idzorek, 2004). For the second method, we use mean-variance optimization, and maximize the Sharpe ratio:

$$
\begin{aligned}
& \max \frac{\mathbf{w}_{B L, t}{ }^{\prime} \boldsymbol{\mu}_{B L, t}}{\sqrt{\mathbf{w}_{B L, t}{ }^{\prime} \mathbf{V}_{t} \mathbf{w}_{B L, t}}} \\
& \text { subject to } \mathbf{w}_{B L, t}{ }^{\prime} \mathbf{1}=1
\end{aligned}
$$

where $\boldsymbol{\mu}_{B L, t}$ is the expected excess return of the BL portfolio in $(8), \sqrt{\mathbf{w}_{B L, t}{ }^{\prime} \mathbf{V}_{t} \mathbf{w}_{B L, t}}$ is the conditional portfolio standard deviation, $\mathbf{w}_{B L, t}$ is the $N \times 1$ vector of portfolio weights and $\mathbf{1}$ is an $N \times 1$ vector of ones. Thus, the vector of optimal weights for the SR-BL portfolio is given by:

$$
\mathbf{w}_{B L, t}^{*}=\frac{\mathbf{V}_{t}^{-1} \boldsymbol{\mu}_{B L, t}}{\mathbf{1}^{\prime} \mathbf{V}_{t}^{-1} \boldsymbol{\mu}_{B L, t}}
$$

In both methods, the standard deviation is used to measure the portfolio risk. As noted above, this is only appropriate when returns are normally distributed and so we consider a more general formulation of the maximal reward-to-risk portfolio, where risk is measured by the $\mathrm{VaR}$ or $\mathrm{CVaR}$ of the $\mathrm{BL}$ portfolio based on the assumption of conditional joint elliptically distributed returns and finite variance (see Ortobelli et al, 2006a and 2006b). In this paper, in order to maintain the simplicity of the estimation of $\mathrm{VaR}$ and $\mathrm{CVaR}$, we consider the cases that returns have either a normal distribution or a $t$-distribution. In particular, the optimization problem with maximal reward-to- $\mathrm{VaR}(\mathrm{MVaR}-\mathrm{BL})$ is:

$$
\begin{gathered}
\max \frac{\mathbf{w}_{B L, t}{ }^{\prime} \boldsymbol{\mu}_{B L, t}}{\operatorname{VaR} R_{\alpha, t}} \\
\text { subject to } \mathbf{w}_{B L, t}{ }^{\prime} \mathbf{1}=1
\end{gathered}
$$


where $\operatorname{VaR}_{\alpha, t}$ is the expected maximum loss on the BL portfolio at time $t$ with a confidence level of $1-\alpha$. The $\mathrm{VaR}$ of the BL portfolio at time $t$ can be expressed as:

$$
\operatorname{VaR}_{\alpha, t}=\xi_{\alpha} \sqrt{\mathbf{w}_{B L, t}{ }^{\prime} \mathbf{V}_{t} \mathbf{w}_{B L, t}}-\mathbf{w}_{B L, t}{ }^{\prime} \boldsymbol{\mu}_{B L, t}
$$

where $\xi_{\alpha}=-F^{-1}(1-\alpha)$ is the $1-\alpha \%$ quantile of the cumulative distribution $F($.$) and \alpha$ is equal to $1 \%, 5 \%$ or $10 \%$ (see, for example, Rockafellar and Uryasev, 2000).

Similarly, the optimization problem with maximal reward-to-CVaR (MCVaR-BL) is:

$$
\begin{gathered}
\max \frac{\mathbf{w}_{B L, t}{ }^{\prime} \boldsymbol{\mu}_{B L, t}}{C V a R_{\alpha, t}} \\
\text { subject to } \mathbf{w}_{B L, t} \mathbf{1}=1
\end{gathered}
$$

where $C V_{a} R_{\alpha, t}$ is the average loss exceeding the expected maximum loss $\operatorname{VaR}_{\alpha, t}$ at time $t$ on the BL portfolio with a certain confidence level of $1-\alpha$. The $\mathrm{CVaR}$ of the BL portfolio at time $t$ is:

$$
C \operatorname{VaR}_{\alpha, t}=\zeta_{\alpha, t} \sqrt{\mathbf{w}_{B L, t}{ }^{\prime} \mathbf{V}_{t} \mathbf{w}_{B L, t}}-\mathbf{w}_{B L, t}{ }^{\prime} \boldsymbol{\mu}_{B L, t}
$$

where $\zeta_{\alpha, t}=\frac{-\int_{-\infty}^{-F^{-1}(1-\alpha)} g f(g) d g}{1-\alpha}$ and $g=-\mathbf{w}_{B L, t}{ }^{\prime} \boldsymbol{\mu}_{B L, t}-V a R_{\alpha, t} \quad$ (see, for example, Rockafellar and Uryasev, 2000). Note that all of our proposed dynamic BL portfolios allow for short sales.

\subsection{The Dynamic Risk-adjusted BL Portfolio}

Giacometti et al. (2007) improve the BL framework by relaxing the assumption of normally distributed returns. They also incorporate alternative risk measures into the calculation of equilibrium returns. However, they do not evaluate the performance of their method in a 
dynamic asset allocation framework, which we address here. The first step is to estimate the time-varying risk-adjusted equilibrium return. The optimization problem is given by:

$$
\max \left(\mathbf{w}_{t}{ }^{\prime} \boldsymbol{\pi}_{t}-\frac{\delta_{t}}{2} \vartheta\left(\mathbf{w}_{t}{ }^{\prime} \mathbf{r}_{t}\right)\right)
$$

where $\vartheta\left(\mathbf{w}_{t}{ }^{\prime} \mathbf{r}_{t}\right)$ indicates the measure of risk (i.e. variance, $\mathrm{VaR}$ or $\left.\mathrm{CVaR}\right)$ of the portfolio return $\mathbf{w}_{t}{ }^{\prime} \mathbf{r}_{t}$ and the equilibrium returns at time $t$ are given by:

$$
\begin{gathered}
\boldsymbol{\pi}_{t}=\delta_{t} \mathbf{H}_{t} \mathbf{w}_{t} \\
\boldsymbol{\pi}_{t}=\frac{\delta_{t}}{2}\left(\operatorname{VaR}_{\alpha, t} \frac{\mathbf{H}_{t} \mathbf{w}_{t}}{\sqrt{\mathbf{w}_{t}{ }^{\prime} \mathbf{H}_{t} \mathbf{w}_{t}}}-E(\mathbf{r})\right) \\
\boldsymbol{\pi}_{t}=\frac{\delta_{t}}{2}\left(C V a R_{\alpha, t} \frac{\mathbf{H}_{t} \mathbf{w}_{t}}{\sqrt{\mathbf{w}_{t}{ }^{\prime} \mathbf{H}_{t} \mathbf{w}_{t}}}-E(\mathbf{r})\right)
\end{gathered}
$$

where $\mathbf{H}_{t}$ is the covariance matrix at time $t$ obtained from the conditional volatility models (2)-(4), under the normal or t-distribution. $\operatorname{VaR}_{\alpha, t}$ and $C V a R_{\alpha, t}$ are the VaR and the CVaR at time $t$ for the corresponding distribution at the $1-\alpha$ confidence level and $E(\mathbf{r})$ is the expected return. Since we have estimated the risk-adjusted equilibrium return $\boldsymbol{\pi}_{t}$, we substitute $\boldsymbol{\pi}_{t}$ in Equation (8) with $\boldsymbol{\pi}_{t}$ in Equations (18) to (20). Then, we use Equation (10) to construct the implied risk-adjusted BL portfolio, and Equation (12) to construct the riskadjusted SR-BL portfolio.

\section{Empirical Results}

In this section, we discuss the out-of-sample performance of the dynamic BL portfolios detailed above. The results of the in-sample analysis are available upon request. Given the weights obtained from the optimization of the unconstrained dynamic BL models and dynamic risk-adjusted BL models, we calculate buy-and-hold returns on the portfolio for a holding period of one month and repeat the calculation until the end of the sample, thus obtaining the time series of realized portfolio returns. We report the average, standard deviation, skewness and kurtosis of the portfolio returns in the out-of-sample period. We also use the average Herfindahl index (AHI) to measure diversification. The higher the value of 
the AHI, the less diversified the portfolio. To evaluate the performance of the portfolio, we use the information ratio, the reward-to-risk ratio (i.e. the Sharpe ratio) and the ratio of reward-to-downside risk, where downside risk is measured by VaR or CVaR computed from the empirical distribution. The information ratio measures the active return of the portfolio divided by the amount of portfolio risk relative to the benchmark. With these evaluation criteria, we compare the risk-adjusted $\mathrm{BL}$ portfolios to the benchmark, the naive $1 / \mathrm{N}$ portfolio, and the unconstrained BL portfolios. Finally, we investigate the impact of the choice of distribution and confidence level on the performance of the dynamic BL portfolios.

Following Giacometti et al. (2007), we initially estimate each of the three volatility models using the first 110 observations (from January 1994 to February 2003) to generate a onemonth-ahead out-of-sample forecast of the conditional covariance matrix for month 111 (March 2003). The estimation sample is then rolled forward by one month, the models are reestimated and used to generate out-of-sample forecasts for month 112, and so on until the end of the sample. For each iteration, the starting parameter values for each model are set to the values estimated in the previous iteration. This procedure results in a total of 151 out-ofsample monthly forecasts. We then construct the momentum portfolio with a holding period of six months to use as the view vector in the BL model. Thus, the first month in which the BL portfolio is formed is August 2003 and so the total number of out-of-sample estimates is reduced to 145 . We report the out-of-sample portfolio performance results in Table 3.

\section{[Table 3]}

Table 3 shows that all the unconstrained BL portfolios outperform both the benchmark portfolio and the $1 / \mathrm{N}$ portfolio, with better Sharpe ratios and reward-to-downside risk ratios. In addition, the unconstrained BL portfolios have significantly lower values of the AHI (less than 1) than the traditional static mean-variance portfolio (which has an AHI value of 12.636) implying higher diversification and more moderate weights. ${ }^{1}$

\subsection{The Benchmark Portfolio and the $1 / \mathrm{N}$ Portfolio}

Panel A of Table 3 indicates that while the 1/N portfolio is more diversified, the benchmark portfolio offers superior performance. In particular, the benchmark portfolio generates a

\footnotetext{
${ }^{1}$ For the traditional static mean-variance method, we use historical returns over the estimation period (from January 1994 to July 2003) to estimate the inputs. The portfolio problem can be expressed as maximisation of the Sharpe Ratio, subject to the constraint that the sum of weights is equal to 1, while short sales are allowed with weights between -1 and 2 . The allocated weights do not change during the evaluation period (from August 2003 to August 2015). The sum of the squared weights is equal to 12.636 .
} 
$1.71 \%$ higher Sharpe ratio and a $0.95 \%$ higher reward-to-downside risk ratio relative to the $1 / \mathrm{N}$ portfolio. This result contradicts findings reported elsewhere in the literature regarding the performance of the $1 / \mathrm{N}$ portfolio relative to other, more sophisticated portfolio strategies (see, for example, DeMiguel et al., 2009).

\subsection{The Dynamic Implied BL Portfolio and the SR-BL Portfolio}

Next, we evaluate the performance of the two unconstrained portfolios, namely the implied BL portfolio and the SR-BL portfolio. We find that the implied BL portfolio constructed with the DCC model achieves the highest information ratio of 0.157 (see Panel B). In addition, this portfolio delivers the highest Sharpe, reward-to-VaR and reward-to-CVaR ratios, of 0.199, 0.078 and 0.074 , respectively. These are considerably higher than the corresponding ratios for the DCC-based SR-BL portfolio (see Panel C). Moreover, the information ratio of the DCCbased SR-BL portfolio is 0.075 , which is less than half of that of the implied BL portfolio. The DCC-based SR-BL portfolio has an AHI value of 0.097 and is thus less diversified than the implied DCC-based BL portfolio, which has an AHI value of 0.094.

We also examine the impact of the choice of the volatility model on the performance of the implied BL portfolio and the SR-BL portfolio. We find that the use of the DCC model leads to superior portfolio performance, regardless of the performance measure. Relative to the RW model, the EWMA model results in better performance for the SR-BL portfolio but worse performance for the implied BL portfolio.

\subsection{The Dynamic MVaR-BL Portfolio}

Panel D of Table 3 reports the results for the unconstrained MVaR-BL strategy. The findings on the impact of the volatility models discussed in section 4.2 are similar. In particular, we find that the DCC-based MVaR-BL portfolio is superior at each confidence level under the tdistribution. Further, the EWMA-based MVaR-BL portfolio is superior in terms of riskadjusted performance but inferior in terms of active portfolio performance relative to the RW-based MVaR-BL portfolio. This finding appears robust to the choice of confidence level and distributional assumption. For example, Panel D1 shows that the DCC-MVaR-BL portfolio outperforms both the EWMA-MVaR-BL and the RW-MVaR-BL portfolios under the $\mathrm{t}$-distribution at the $99 \%$ confidence level. Indeed, it achieves the highest information ratio $(0.137)$, Sharpe ratio $(0.200)$, reward-to-VaR ratio $(0.080)$, and reward-to-CVaR ratio (0.067). However, with the highest AHI value (of 0.161), the outperformance comes at the 
cost of lower diversification. Similar conclusions can be drawn at lower confidence levels (Panels D2 and D3).

Next, we assess the impact of the confidence level and distributional assumption on portfolio performance. The EWMA-MVaR-BL and RW-MVaR-BL portfolios appear insensitive to the choice of confidence level and distribution. However, the DCC-MVaR-BL portfolio performance is more sensitive, and performs better at the $99 \%$ confidence level under both the normal and t-distribution (see rows 1 and 4 of Panels D1 to D3). The DCC-MVaR-BL portfolio achieves a slightly better performance under the t-distribution than under the normal distribution at the 99\% confidence level (Panel D1). However, the better performance comes at the cost of lower diversification. Similar conclusions can be drawn for the lower confidence levels (Panels D2 and D3).

\subsection{The Dynamic MCVaR-BL Portfolio}

The finding that the unconstrained MCVaR-BL portfolio with a DCC model is superior to the same portfolio constructed with other volatility models is robust to different confidence levels and distributional assumptions (Panel F). For example, the DCC-MCVaR-BL portfolio achieves higher Sharpe and reward-to-downside risk ratios than the EWMA-based MCVaRBL portfolio under a t-distribution and at the $99 \%$ confidence level (Panel F1). The AHI value of 0.164 implies that the outperformance of the DCC-MCVaR-BL portfolio comes at the cost of lower diversification. Similar conclusions can be drawn under normality and lower confidence levels (Panels F1 to F3).

Turning to the impact of the confidence level and distributional assumption, we see that the performance of the DCC-MCVaR-BL portfolio is better under the t-distribution than under the normal distribution at the $99 \%$ confidence level, but seems insensitive to the choice of distribution at lower confidence levels. Further, the performance of the MCVaR-BL portfolios based on the EWMA and RW models does not appear to be sensitive to the choice of confidence level or distribution.

\subsection{The Dynamic Risk-adjusted BL Portfolio}

In this section, we discuss the performance of the risk-adjusted BL portfolios reported in Table 4.

[Table 4] 


\subsubsection{The Variance-Adjusted BL Portfolio}

The variance-adjusted SR-BL portfolio performs much worse than the implied varianceadjusted BL portfolio, with performance measures that are lower by more than two thirds (Columns 8 to 11, Panels $\mathrm{A}$ and $\mathrm{B}$ of Table 4). This arises from the extreme negative skewness and high kurtosis of the variance-adjusted SR-BL portfolio, leading to higher tail risk.

\subsubsection{The VaR-Adjusted SR-BL Portfolio}

Panel $\mathrm{C}$ of Table 4 indicates that the distributional assumption has a significant impact on the performance of the VaR-adjusted SR-BL portfolio at each confidence level. The t-distribution is better suited to the VaR-adjusted SR-BL portfolio for both performance and diversification. At each confidence level, the Sharpe ratio and reward-to-downside risk ratios under the t-distribution are larger than under the normal distribution. Further, the AHI value under the t-distribution is at least 0.12 lower. Secondly, as the confidence level decreases, the risk-adjusted performance of the VaR-adjusted SR-BL portfolio improves under the normal distribution, while its performance deteriorates slightly under the t-distribution (Rows 1 and 2, Panels C1 to C3).

\subsubsection{The CVaR-Adjusted SR-BL Portfolio}

Panel D of Table 4 indicates that the confidence levels and distributional assumption have a moderate impact on the CVaR-adjusted SR-BL portfolio performance. As the confidence level is reduced from $99 \%$ to $90 \%$, the Sharpe ratio falls from 0.183 to 0.165 , while at the same time, the portfolio becomes more concentrated as suggested by the AHI, which increases from 0.425 to 0.431 . A similar trend of deteriorating performance and diversification as the confidence level is reduced obtains under the t-distribution. Moreover, using the t-distribution rather than the normal distribution, both the diversification and riskadjusted performance of the CVaR-adjusted BL portfolio performance improves. At the $99 \%$ confidence level under the t-distribution, the Sharpe ratio and the reward-to-downside risk ratios of the $\mathrm{CVaR}$-adjusted $\mathrm{BL}$ portfolio are, respectively, $0.4 \%$ and $0.1 \%$ higher than under the normal distribution. The AHI under the t-distribution is about $4.4 \%$ lower than under the normal distribution. At lower confidence levels, the Sharpe ratio and the reward-to-downside risk ratios of the $\mathrm{CVaR}$-adjusted $\mathrm{BL}$ portfolio are $1.4 \%$ higher on average than under the normal distribution. 


\subsection{Dynamic BL Portfolio Performance Ranking}

In this section, we discuss the performance ranking of the dynamic BL portfolios reported in Table 5 .

\section{[Table 5]}

We select the outperforming DCC-based dynamic BL portfolios in each strategy and report their rankings based on different performance measures. Based on the AHI, the $1 / \mathrm{N}$ and the benchmark portfolios have the best performance. However, these two portfolios are outperformed by most dynamic BL portfolios in terms of risk-adjusted performance and active performance. Table 5 shows that the best performing dynamic BL portfolios are the implied BL, the MVaR-BL, MCVaR-BL and the implied variance-adjusted BL portfolios. The implied BL portfolio offers the best active performance with information and reward-to$\mathrm{CVaR}$ ratios of 0.158 and 0.074 respectively. The MVaR-BL shows balanced performance. Overall, the MCVaR-BL portfolio under the t-distribution at the $99 \%$ confidence level performs better than any other portfolio. Although the implied variance-adjusted BL portfolio has the highest reward-to-VaR ratio (0.088) and second highest Sharpe ratio (0.206), its AHI value is nearly twice as large as that of the MCVaR-BL portfolio. All risk-adjusted BL portfolios have much higher AHI values, ranging from 0.307 to 0.541 . Moreover, the CVaRadjusted BL portfolio outperforms the VaR-adjusted BL portfolio. Occasionally, both the VaR- and the CVaR-adjusted BL portfolios perform better than the variance-adjusted BL portfolio, but they do not outperform the unconstrained DCC-BL portfolios.

Finally, our results highlight the importance of using an appropriate reward-to-risk ratio in portfolio performance evaluation (see also Alexander and Baptista, 2003). The performance ranking obtained by the Sharpe ratio under non-normality can be misleading. In this case, the reward-to-downside risk ratios should be used as complementary performance criteria.

\subsection{Summary of the Findings}

The out-of-sample analysis highlights the following important findings. First, the dynamic BL portfolios outperform both the $1 / \mathrm{N}$ and the benchmark portfolios. In addition, the dynamic BL portfolios are more diversified than the mean-variance portfolio. Further, the DCC-based dynamic BL portfolios outperform the EWMA and RW-based portfolios in most cases. Second, the implied BL portfolio outperforms the SR-BL portfolio when using the DCC model and the RW model. Third, both the MVaR-BL and MCVaR-BL portfolios outperform the SR-BL portfolios at high levels of confidence. Further, the MCVaR-BL 
portfolio outperforms the MVaR-BL portfolio and the implied BL portfolio, particularly under the t-distribution and at the 99\% confidence level. Fourth, the MVaR-BL and the MCVaR-BL portfolios constructed with a DCC model perform better under the t-distribution than under the normal distribution. However, there is not a consistent ranking of the riskadjusted portfolio performance and the active portfolio performance, which depends on the volatility model employed. Fifth, the implied variance-adjusted BL portfolio cannot beat the DCC-based MCVaR-BL portfolio under the t-distribution and at the $99 \%$ confidence level; however, it outperforms most of the unconstrained BL portfolios. It also has the best riskadjusted performance and active portfolio performance of the risk-adjusted BL portfolios.

\section{Robustness Checks}

In this section we examine the robustness of our results to the estimation window length and data frequency. We also analyse the stability of our results by considering the sub-period performance of the asset allocation models.

\subsection{Estimation Window and Data Frequency}

We consider the performance of the models using different window lengths and different data frequencies to estimate the model parameters. In particular, Table 6 reports results for the performance of a selection of the DCC-based dynamic portfolios considered in Table 5 (the implied BL, MVaR 0.99t, MCVaR 0.99t, implied variance-adjusted BL, VaR-adjusted BL, and CVaR-adjusted BL portfolios) for estimation window lengths of 36, 60 and 110 months, using both monthly and weekly data. The table also reports results for the benchmark and 1/N portfolios. The superior performance of the dynamic BL portfolios is generally robust to changes in both the estimation window length and the choice of data frequency. All of the risk-adjusted portfolios outperform the benchmark and 1/N portfolios, while the implied-BL portfolio, the MVaR portfolio and the MCVaR portfolio are more sensitive to the changes in the sample size and data frequency, but nevertheless outperform the benchmark and $1 / \mathrm{N}$ portfolios in most cases. The relationship between performance and window length depends on the frequency of the data, reflecting a trade-off between the relevance of the estimation sample in the presence of structural breaks (which is best achieved by using a relatively short sample) and statistical precision (which increases with the sample size). In particular, using monthly and quarterly data, the out-of-sample performance of the dynamic BL portfolios deteriorates as the window length becomes shorter, while with weekly data, the performance of the dynamic BL portfolios tends to improve as the window length becomes shorter. The 
best performance is obtained using weekly data with a window length of 36 months. ${ }^{2}$ Our results are consistent with Bessler et al. (2015), who show that owing to the relatively slow reaction of asset allocation models to structural breaks, the use of long estimation windows (especially those of 48 months and longer) tends to reduce out-of-sample performance.

[Table 6]

\subsection{Sub-period performance}

We divide the full evaluation period (from August 2003 to August 2015) into three subperiods: the pre-financial crisis sub-period (August 2003 to October 2007), the financial crisis sub-period (November 2007 to February 2009) and the post-financial crisis sub-period (March 2009 to August 2015). We then assess the performance of the BL portfolios in each of the three sub-periods. Owing to the reduced sample size, and in view of the findings reported above, we use weekly data and an estimation window of 36 months. Table 7 reports the results of the sub-period analysis using the DCC-based dynamic BL portfolios in each strategy. Consistent with our results for the full evaluation period, all of the dynamic BL portfolios outperform the benchmark and $1 / \mathrm{N}$ portfolios in both the financial crisis sub-period and the post-financial crisis sub-period. In the pre-financial crisis sub-period, most of the risk-adjusted BL portfolios outperform the benchmark and $1 / \mathrm{N}$ portfolios. However, the relative performance of the different strategies is time-dependent. In the financial crisis subperiod, the implied BL portfolio, the SR-BL portfolio, the MVaR portfolio and the MCVaR portfolio outperform the risk-adjusted BL portfolios, with positive Sharpe ratios and rewardto-downside risk ratios. However, in the pre-financial crisis and post-financial crisis subperiods, the risk-adjusted BL portfolios perform better than the other strategies.

\section{[Table 7]}

\section{Conclusion}

In this paper, we extend the Black-Litterman methodology to a dynamic asset allocation framework. The main contribution of this paper is to relax the assumption of a constant conditional distribution of returns in the BL model. Moreover, this paper also improves the BL framework by using alternative risk measures (VaR and $\mathrm{CVaR}$ ) under different distributional assumptions (normal and t-distribution). The dynamic unconstrained $\mathrm{BL}$ portfolio nests the implied BL portfolio formed by reverse optimization of the BL model, the

\footnotetext{
${ }^{2}$ Detailed results for the full set of portfolios are available on request.
} 
SR-BL portfolio with maximal Sharpe ratio, the MVaR-BL portfolio with maximal rewardto-VaR ratio and the MCVaR-BL portfolio with maximal reward-to-CVaR ratio. We analyse four risk-adjusted BL portfolios, the implied variance-adjusted BL portfolio, varianceadjusted SR-BL portfolio, VaR-adjusted SR-BL portfolio and CVaR-adjusted SR-BL portfolio. We also use different performance measures, which lead to different rankings of these BL portfolios. Further, we examine the effect of the choice of volatility model, distributional assumption and the specified confidence level on portfolio diversification and portfolio performance.

The out-of-sample analysis suggests that the dynamic BL asset allocation framework performs well. In particular, the dynamic BL portfolios and risk-adjusted BL portfolios outperform the benchmark and the $1 / \mathrm{N}$ portfolio and lead to more diversified portfolios relative to the standard mean-variance portfolio. We find that the DCC model is a suitable volatility model choice when constructing a dynamic BL portfolio. Since the Sharpe ratio is not an appropriate performance ratio when asset returns are non-normal, alternative performance ratios (reward-to- $\mathrm{VaR}$ and reward-to-CVaR ratios) are used to construct unconstrained BL portfolios and evaluate portfolio performance. The alternative is to use the estimated risk-adjusted equilibrium returns in the dynamic BL asset allocation process, although this leads to much more concentrated portfolios relative to the unconstrained $\mathrm{BL}$ portfolios.

The dynamic BL asset allocation approach could be extended in several directions. First, it could be examined under the new performance measures of Biglova et al. (2004) and Rachev et al. (2007). In particular, it would be interesting to investigate the performance of the portfolios that optimize the Rachev ratio and Rachev generalised ratio. In addition, adding tracking error constraints to improve the active portfolio performance following the method of Palomba (2008) is another direction for future research. It would also be useful to investigate the performance of the dynamic BL portfolio in other markets, such as bonds, currencies and commodities, and over a longer sample period. 


\section{REFERENCES}

ALEXANDER, G.J. and BAPTISTA, A.M. (2003) Portfolio performance evaluation using value at risk. The Journal of Portfolio Management, 29 (4), 93-102.

ALEXANDER, C. O. \& LEIGH, C. T. (1997) On the covariance matrices used in value at risk models. The Journal of Derivatives, 4, 50-62.

ALEXANDER, C. (1998). Volatility and correlation: measurement, models and applications. Risk Management and Analysis, 1, 125-171.

ANDERSEN, T. G., BOLlERSLEV, T. \& DIEBOLD, F. X. (2004) Parametric and nonparametric measurements of volatility. In: Aït-Sahalia, Y., Hansen, L. (Eds.), Handbook of Financial Econometrics. North-Holland, Amsterdam.

ANG, A. \& CHEN, J. (2002) Asymmetric correlations of equity portfolios. Journal of Financial Economics, 63 (3), 443-494.

ARTZNER, P., HEATH, D., DELBAEN, F. \& EBER, J. (1999) Coherent Measures of Risk. Mathematical Finance, 9, 203-228.

BABAMETO, E. \& HARRIS, R. (2009) Exploiting predictability in the returns to value and momentum investment strategies: A portfolio approach. Professional Investor, June, 39-43.

BEACH, S. \& ORLOV, A. (2007) An application of the Black-Litterman model with EGARCH-M-derived views for international portfolio management. Financial Markets and Portfolio Management, 21, 147-166.

BERTSIMAS, D., GUPTA, V. \& PASCHALIDIS, I.C. (2012) Inverse optimization: a new perspective on the Black-Litterman model. Operations Research, 60 (6), 1389-1403.

BESSLER, W., OPFER, H. \& WOLFF, D. (2014) Multi-asset portfolio optimization and outof-sample performance: an evaluation of Black-Litterman, mean-variance, and naïve diversification approaches. The European Journal of Finance, 1-30.

BEST, M. \& GRAUER, R. (1991) On the sensitivity of mean-variance-efficient portfolios to changes in asset means: some analytical and computational results. Review of Financial Studies, 4, 315-342.

BEVAN, A., \& WINKELMANN, K. (1998) Using the Black-Litterman global asset allocation model: three years of practical experience. Fixed Income Research, Goldman Sachs \& Company, New York.

BIGlOVA, A., ORTOBElli, S., RACHEV, S. T. \& STOYANOV, S. (2004) Different approaches to risk estimation in portfolio theory. The Journal of Portfolio Management, 31, 103-112.

BLACK, F. \& LITTERMAN, R. (1991) Asset allocation: combining investor views with market equilibrium. Journal of Fixed Income, 1 (1), 7-18. 
BLACK, F. \& LITTERMAN, R. (1992) Global portfolio optimisation. Financial Analysts Journal, 48, 28-43.

BOLLERSLEV, T., ENGLE, R. F. \& WOOLDRIDGE, J. M. (1988) A Capital Asset Pricing Model with time-varying covariances. The Journal of Political Economy, 96, 116-131.

BOUDOUKH, J., RICHARDSON, M. \& WHITELAW, R. F. (1997) Investigation of a class of volatility estimators. The Journal of Derivatives, 4, 63-71.

BRANDT, M. W. \& WANG, K. Q. (2003) Time-varying risk aversion and unexpected inflation. Journal of Monetary Economics, 50, 1457-1498.

CHIARAWONGSE, A., KIATSUPAIBUL, S., TIRAPAT, S. \& VAN ROY, B. (2012) Portfolio selection with qualitative input. Journal of Banking \& Finance, 36, 489-496.

DEMIGUEL, V., GARLAPPI, L. \& UPPAL, R. (2009) Optimal versus naive diversification: How inefficient is the 1/N portfolio strategy? Review of Financial Studies, 22 (5), 1915-1953.

ENGLE, R. F. (1982) Autoregressive conditional heteroscedasticity with estimates of the variance of United Kingdom inflation. Econometrica, 50, 987-1007.

ENGLE, R. F. (2002). 'Dynamic Conditional Correlation: a simple class of multivariate generalized autoregressive conditional heteroskedasticity models', Journal of Business and Economic Statistics, 20, 339-350.

ENGLE, R. F. \& SHEPPARD, K. (2001) Theoretical and empirical properties of dynamic conditional correlation multivariate GARCH. NBER Working Paper No. W8554, Available at SSRN.

FABOZZI, F. J., FOCARDI, S. M. \& KOLM, P. N. (2006) Incorporating trading strategies in the Black-Litterman framework. The Journal of Trading, 1, 28-37.

FARINELLI, S., FERREIRA, M., ROSSELLO, D., THOENY, M. \& TIBILETTI, L. (2008) Beyond Sharpe ratio: Optimal asset allocation using different performance ratios. Journal of Banking \& Finance, 32, 2057-2063.

FARINELLI, S., FERREIRA, M., ROSSELLO, D., THOENY, M. \& TIBILETTI, L. (2009) Optimal asset allocation aid system: From "one-size" vs "tailor-made" performance ratio. European Journal of Operational Research, 192, 209-215.

FARINELLI, S. \& TIBILETTI, L. (2008) Sharpe thinking in asset ranking with one-sided measures. European Journal of Operational Research, 185, 1542-1547.

FAVRE, L. \& GALEANO, J.A. (2002) Mean-modified Value at Risk optimization with hedge funds, Journal of Alternative Investment, 5(2), 21-25.

FERNANDES, J. L. B., ORNELAS, J. R. H. \& CUSICANQUI, O. A. M. (2011) Combining equilibrium, resampling and analyst's views in portfolio optimisation. Journal of Banking \& Finance, 36, 1354-1361. 
GIACOMETTI, R., BERTOCCHI, M., RACHEV, S. T. \& FABOZZI, F. J. (2007) Stable distributions in the Black-Litterman approach to asset allocation. Quantitative Finance, 7, 423-433.

GOH, J.W., LIM, K.G., SIM, M. \& ZHANG, W. (2012) Portfolio value-at-risk optimization for asymmetrically distributed asset returns. European Journal of Operational Research, 221 (2), 397-406.

GRAUER, R. R. \& SHEN, F. C. (2000) Do constraints improve portfolio performance? Journal of Banking \& Finance, 24, 1253-1274.

GREEN, R. C. \& HOLLIFIELD, B. (1992) When will mean-variance efficient porfolios be well diversified. Journal of Finance, 47(5), 1785-1810.

GUERMAT, C. \& HARRIS, R. D. (2002) Robust conditional variance estimation and valueat-risk. Journal of Risk, 4, 25-42.

HARVEY, C.R., LIECHTY, J.C., LIECHTY, M.W. \& MÜLLER, P. (2010) Portfolio selection with higher moments. Quantitative Finance, 10 (5), 469-485.

HEROLD, U. \& MAURER, R. (2003) Bayesian asset allocation and US domestic bias. Financial Analysts Journal, 59, 54-65.

HUANG, D., ZHU, S., FABOZZI, F.J. \& FUKUSHIMA, M. (2010) Portfolio selection under distributional uncertainty: A relative robust $\mathrm{CVaR}$ approach. European Journal of Operational Research, 203 (1), 185-194.

IDZOREK, T. (2004) A step-by-step guide to the Black-Litterman model. Zephyr Working Document.

JAGANNATHAN, R., \& MA, T. (2003) Risk reduction in large portfolios: Why imposing the wrong constraints helps. Journal of Finance, 58, 1651 - 1684.

JORION, P. (1991) Bayesian and CAPM estimators of the means: Implications for portfolio selection. Journal of Banking \& Finance, 15 (3), 717-727.

J. P. MORGAN (1994) RiskMetrics technical document. New York: Morgan Guaranty Trust Company.

KALOTYCHOU, E., STAIKOURAS, S.K. \& ZHAO, G. (2014) The role of correlation dynamics in sector allocation. Journal of Banking \& Finance, 48, 1-12.

KERSTENS, K., MOUNIR, A. \& VAN DE WOESTYNE, I. (2011) Geometric representation of the mean-variance-skewness portfolio frontier based upon the shortage function. European Journal of Operational Research, 210 (1), 81-94.

KOLM, P.N., TÜTÜNCÜ, R. \& FABOZZI, F.J. (2014) 60 Years of portfolio optimization: Practical challenges and current trends. European Journal of Operational Research, 234 (2), 356-371. 
MARKOWITZ, H. (1952) Portfolio selection. Journal of Finance, 7 (1), 77-91.

MARTELLINI, L. \& ZIEMANN, V. (2007) Extending Black-Litterman analysis beyond the mean-variance framework. The Journal of Portfolio Management, 33 (4), 33-44.

MEUCCI, A. (2009) Risk and asset allocation, Springer.

MITTON, T. \& VORKINK, K. (2007) Equilibrium underdiversification and the preference for skewness. Review of Financial Studies, 20 (4), 1255-1288.

ORTOBELLI, S., LAMANTIA, F. \& RACHEV, S. (2006a) VaR, CVaR and time rules with elliptical and asymmetric stable distributed returns. Investment Management and Financial Innovations 3(4), 19-39

ORTOBELLI, S., LAMANTIA, F. \& RACHEV, S. (2006b) An empirical comparison among $\mathrm{VaR}$ models and time rules with elliptical and stable distributed returns. Investment Management and Financial Innovations 3(3), 8-29

PALOMBA, G. (2008) Multivariate GARCH models and the Black-Litterman approach for tracking error constrained portfolios: an empirical analysis. Global Business and Economics Review, 10, 379-413.

PEIRO, A. (1999) Skewness in financial returns. Journal of Banking \& Finance, 23, 847862.

RACHEV, S., MARTIN, D. \& SIBOULET, F. (2003) Phi-alpha optimal portfolios and Extreme Risk Management, Wilmott Magazine of Finance, November, pp. 70-83.

RACHEV, S., JAŠIĆ, T., STOYANOV, S. \& FABOZZI, F. J. (2007) Momentum strategies based on reward-risk stock selection criteria. Journal of Banking \& Finance, 31, 2325-2346.

RICHARD, A. J. (1997) Winner-loser reversals in national stock market indices: can they be explained? Journal of Finance, 52, 2129-2144.

ROCKAFELLAR, R. \& URYASEV, S. (2000) Optimisation of conditional value-at-risk. Journal of Risk, 2, 21-42.

ROCKAFELLAR, R.T. \& URYASEV, S. (2002) Conditional value-at-risk for general loss distributions. Journal of Banking \& Finance, 26 (7), 1443-1471.

SCOTT, R.C. \& HORVATH, P.A. (1980) On the direction of preference for moments of higher order than the variance. Journal of Finance, 35, 915-919.

STINSTRA, E. \& DEN HERTOG, D. (2008) Robust optimization using computer experiments. European Journal of Operational Research, 191 (3), 816-837.

TÜTÜNCÜ, R.H. \& KOENIG, M. (2004) Robust asset allocation. Annals of Operations Research, 132 (1-4), 157-187. 


\section{Table 1 Summary Statistics for the FTSE Sector Indices Excess Returns}

Table 1 reports summary statistics for the monthly excess return series on 10 FTSE Sector Indices in UK, US and Japan countries for the period January 1994 to August 2015. The table also reports the statistic and p-value of the Jarque-Bera test of the null hypothesis that the series are normally distributed distribution.

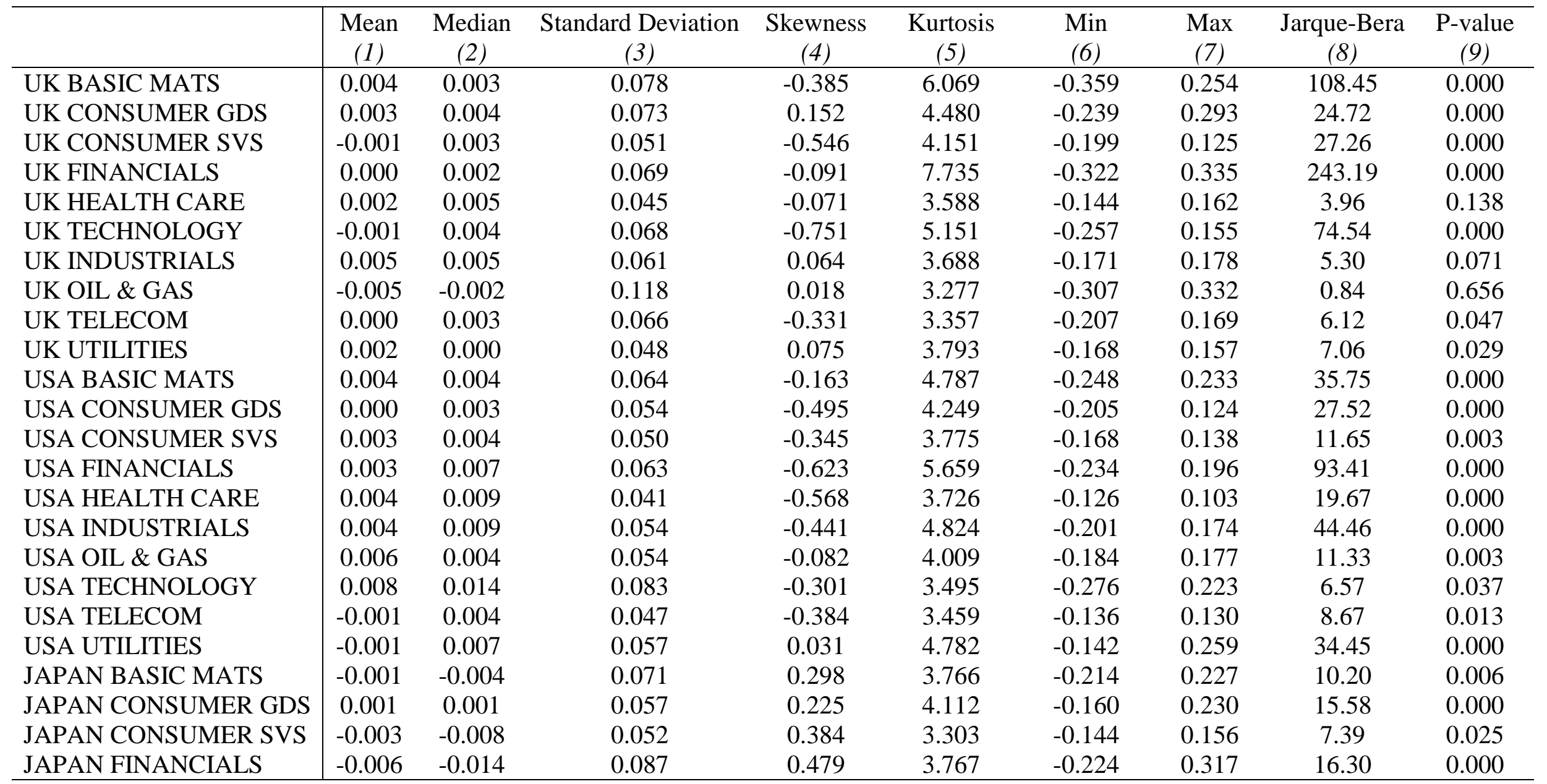

Table 1 (continued) 
Table 1 (continued)

\begin{tabular}{l|ccccccccc}
\hline & $\begin{array}{c}\text { Mean } \\
(1)\end{array}$ & $\begin{array}{c}\text { Median } \\
(2)\end{array}$ & $\begin{array}{c}\text { Standard Deviation } \\
(3)\end{array}$ & $\begin{array}{c}\text { Skewness } \\
(4)\end{array}$ & $\begin{array}{c}\text { Kurtosis } \\
(5)\end{array}$ & $\begin{array}{c}\text { Min } \\
(6)\end{array}$ & $\begin{array}{c}\text { Max } \\
(7)\end{array}$ & $\begin{array}{c}\text { Jarque-Bera } \\
(8)\end{array}$ & $\begin{array}{c}\text { P-value } \\
(9)\end{array}$ \\
\hline JAPAN HEALTH CARE & 0.000 & 0.000 & 0.051 & 0.430 & 4.726 & -0.152 & 0.218 & 40.29 & 0.000 \\
JAPAN INDUSTRIALS & 0.001 & 0.005 & 0.062 & -0.107 & 3.089 & -0.193 & 0.179 & 0.58 & 0.748 \\
JAPAN OIL \& GAS & -0.001 & -0.005 & 0.086 & 0.095 & 3.763 & -0.271 & 0.271 & 6.70 & 0.035 \\
JAPAN TECHNOLOGY & 0.001 & -0.003 & 0.087 & 0.296 & 3.376 & -0.197 & 0.293 & 5.32 & 0.070 \\
JAPAN TELECOM & 0.000 & -0.003 & 0.079 & 0.855 & 6.032 & -0.230 & 0.375 & 131.27 & 0.000 \\
JAPAN UTILITIES & -0.002 & -0.006 & 0.052 & 0.428 & 4.372 & -0.117 & 0.231 & 28.32 & 0.000 \\
\hline
\end{tabular}




\section{Table 2 Time Series Properties}

Table 2 reports the test statistics for autocorrelation, autoregressive conditional heteroskedasticity (ARCH) and dynamic conditional correlation for the full sample from January 1994 to August 2015. The Ljung-Box-Q test statistic for autocorrelation of up to order 10 is asymptotically distributed as a central Chi-square with 10 d.o.f. The ARCH (1) statistic is asymptotically distributed as a central Chi-square with one d.o.f. The DCC statistic is distributed as a central Chi-square with one d.o.f. *, ** and *** denote significance at $10 \%, 5 \%$ and $1 \%$ levels respectively. In the DCC test, ' 30 assets' refers to the sample that includes all assets, and ' 18 assets' refers to the sample that includes assets selected with significant autocorrelation in the squared residuals with one lag.

\begin{tabular}{|c|c|c|c|c|c|c|c|}
\hline & $\begin{array}{c}\mathrm{ACF}(1) \\
(1)\end{array}$ & $\begin{array}{c}\mathrm{ACF}(2) \\
(2)\end{array}$ & $\begin{array}{c}\mathrm{ACF}(3) \\
(3)\end{array}$ & $\begin{array}{c}\mathrm{ACF}(4) \\
(4)\end{array}$ & $\begin{array}{c}\mathrm{ACF}(5) \\
(5)\end{array}$ & $\begin{array}{c}\text { LB-Q(10) } \\
(6)\end{array}$ & $\begin{array}{c}\mathrm{ARCH}(1) \\
(7)\end{array}$ \\
\hline UK CONSUMER GDS & -0.034 & -0.001 & 0.093 & -0.120 & -0.056 & $18.377^{*}$ & 0.021 \\
\hline UK FINANCIALS & $0.165 * * *$ & $0.029 *$ & $0.090 *$ & $0.070 *$ & $-0.033^{*}$ & 17.040 & $11.760 * * *$ \\
\hline UK HEALTH CARE & -0.075 & 0.059 & 0.009 & -0.094 & -0.005 & 5.758 & 1.319 \\
\hline UK TECHNOLOGY & 0.070 & -0.009 & -0.001 & 0.032 & 0.028 & 5.662 & 0.268 \\
\hline UK TELECOM & 0.093 & -0.018 & $0.189 * * *$ & $-0.007 *$ & $0.071^{*}$ & 17.808 & $7.963 * * *$ \\
\hline UK UTILITIES & 0.020 & 0.066 & 0.034 & 0.052 & -0.068 & 10.341 & $4.160^{*}$ \\
\hline USA BASIC MATS & 0.021 & 0.041 & 0.044 & 0.003 & -0.058 & 13.554 & $28.250 * * *$ \\
\hline USA CONSUMER GDS & 0.027 & -0.146 & 0.008 & 0.008 & 0.006 & 11.018 & 1.458 \\
\hline USA CONSUMER SVS & 0.063 & -0.113 & 0.070 & 0.010 & -0.024 & 6.323 & $7.070 * * *$ \\
\hline USA FINANCIALS & 0.082 & -0.033 & 0.067 & 0.073 & 0.071 & 14.632 & $17.591 * * *$ \\
\hline USA UTILITIES & 0.005 & -0.049 & 0.085 & 0.013 & 0.081 & 13.521 & $13.540 * * *$ \\
\hline JAPAN BASIC MATS & 0.083 & 0.005 & 0.095 & -0.027 & 0.070 & 11.657 & $19.241 * * *$ \\
\hline JAPAN CONSUMER GDS & 0.063 & -0.028 & $0.158^{*}$ & -0.006 & 0.025 & 15.853 & 1.574 \\
\hline JAPAN CONSUMER SVS & 0.083 & -0.089 & 0.072 & -0.107 & 0.029 & 14.931 & 0.741 \\
\hline JAPAN FINANCIALS & 0.072 & -0.041 & 0.079 & -0.029 & 0.031 & 9.811 & 0.043 \\
\hline
\end{tabular}

Table 2 (continued) 
Table 2 (continued)

\begin{tabular}{|c|c|c|c|c|c|c|c|}
\hline & $\begin{array}{c}\mathrm{ACF}(1) \\
(1)\end{array}$ & $\begin{array}{c}\mathrm{ACF}(2) \\
(2)\end{array}$ & $\begin{array}{c}\mathrm{ACF}(3) \\
(3)\end{array}$ & $\begin{array}{c}\mathrm{ACF}(4) \\
(4)\end{array}$ & $\begin{array}{c}\mathrm{ACF}(5) \\
(5)\end{array}$ & $\begin{array}{c}\mathrm{LB}-\mathrm{Q}(10) \\
(6)\end{array}$ & $\begin{array}{c}\mathrm{ARCH}(1) \\
(7)\end{array}$ \\
\hline JAPAN INDUSTRIALS & $0.161 * * *$ & $0.009 * *$ & $0.123 * * *$ & $-0.024 * *$ & $0.012 *$ & 14.524 & $7.407 * * *$ \\
\hline JAPAN TECHNOLOGY & $0.124 *$ & $0.114^{* *}$ & $0.176^{* * *}$ & $0.039 * * *$ & $0.068 * * *$ & $23.638 * *$ & $32.434 * * *$ \\
\hline JAPAN TELECOM & $0.180 * * *$ & $0.038 * * *$ & $0.055^{* *}$ & $0.000 * * *$ & $0.152 * * *$ & $21.111^{*}$ & $12.101 * * *$ \\
\hline JAPAN UTILITIES & -0.068 & 0.067 & -0.111 & -0.041 & -0.046 & 8.596 & 0.764 \\
\hline
\end{tabular}

\begin{tabular}{l|cc}
\hline DCC test & statistic & p-Value \\
\hline 30 Assets & 3.280 & 0.194 \\
18 Assets & 11.911 & 0.003 \\
\hline
\end{tabular}




\section{Table 3 Out-of-sample Unconstrained BL Portfolio Performance}

Table 3 reports realized unconstrained BL portfolio performance compared with the benchmark performance in the period from August 2003 to August 2015. Return is the average realized excess return, Sharpe Ratio (SR) is the average excess realized return divided by the standard deviation (SD). Average Herfindahl index (AHI) is the average value of the time-varying Herfindahl index. Information Ratio (IR) is the average active return divided by the standard deviation of active return. Both $\mathrm{VaR}$ and $\mathrm{CVaR}$ are measured using the empirical distribution. $\mu / \mathrm{VaR}$ and $\mu / C V a R$ evaluate the excess return per unit of tail risk. In the construction of the portfolio, both VaR and CVaR are estimated by the parametric method with the assumption of a normal distribution (' $N$ ') or $t$-distribution (' $t$ ') at confidence levels of $99 \%, 95 \%$ and $90 \%$. The implied BL portfolio is constructed by reverse optimization of the utility function. The SR-BL portfolio is constructed by maximizing the SR in. The MVaR-BL portfolio is constructed by maximizing the reward-to-VaR ratio. The MCVaR-BL portfolio is constructed by maximizing the reward-to-CVaR ratio.

\begin{tabular}{|c|c|c|c|c|c|c|c|c|c|c|c|c|}
\hline & & $\begin{array}{c}\text { Return } \\
(1)\end{array}$ & $\begin{array}{l}\text { SD } \\
(2) \\
\end{array}$ & $\begin{array}{c}\text { Skewness } \\
\text { (3) }\end{array}$ & $\begin{array}{c}\text { Kurtosis } \\
(4)\end{array}$ & $\begin{array}{c}\mathrm{VaR} \\
(5) \\
\end{array}$ & $\begin{array}{c}\text { CVaR } \\
(6) \\
\end{array}$ & $\begin{array}{c}\text { AHI } \\
(7)\end{array}$ & $\begin{array}{l}\text { IR } \\
(8) \\
\end{array}$ & $\begin{array}{l}\text { SR } \\
(9) \\
\end{array}$ & $\begin{array}{c}\mu / \operatorname{VaR} \\
(10) \\
\end{array}$ & $\begin{array}{c}\mu / C V a R \\
(11)\end{array}$ \\
\hline \multicolumn{13}{|c|}{ Panel A. The benchmark portfolio and the $1 / N$ portfolio } \\
\hline Benchmark & & 0.005 & 0.039 & -0.855 & 5.487 & 0.107 & 0.149 & 0.075 & $\mathrm{NaN}$ & 0.135 & 0.049 & 0.036 \\
\hline \multicolumn{13}{|c|}{ Panel B. The implied BL portfolio } \\
\hline Implied BL & DCC & 0.008 & 0.042 & -0.230 & 4.201 & 0.107 & 0.112 & 0.094 & 0.157 & 0.199 & 0.078 & 0.074 \\
\hline \multicolumn{13}{|c|}{ Panel C. The SR-BL portfolio } \\
\hline \multirow{3}{*}{ SR-BL } & DCC & 0.007 & 0.042 & -0.628 & 5.599 & 0.109 & 0.151 & 0.097 & 0.075 & 0.164 & 0.063 & 0.045 \\
\hline & EWMA & 0.006 & 0.038 & -0.231 & 4.121 & 0.093 & 0.101 & 0.098 & 0.072 & 0.161 & 0.067 & 0.061 \\
\hline & RW & 0.006 & 0.039 & -0.462 & 4.716 & 0.111 & 0.123 & 0.103 & 0.038 & 0.145 & 0.052 & 0.047 \\
\hline
\end{tabular}

\section{Table 3 (continued)}


Table 3 (continued)

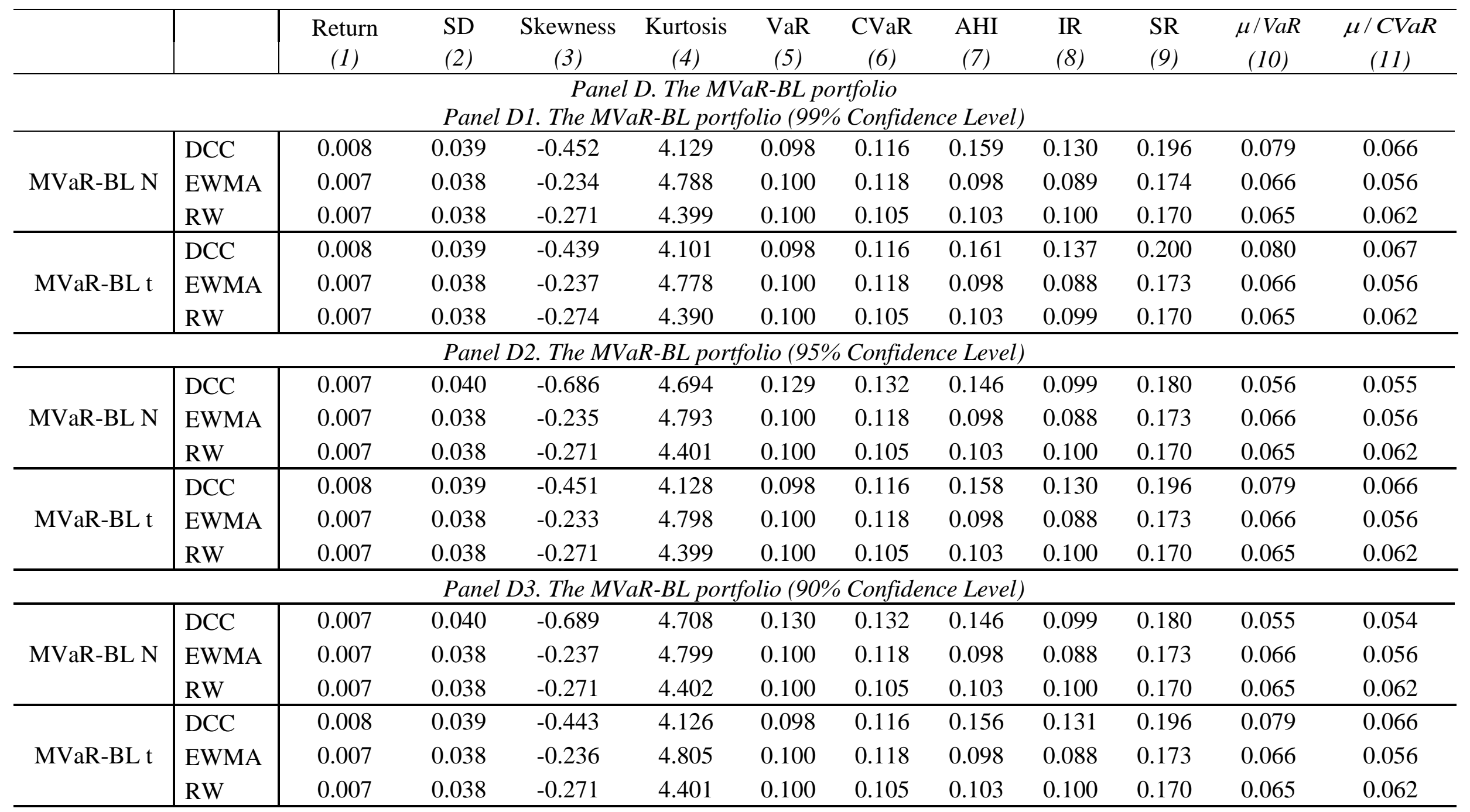

Table 3 (continued) 
Table 3 (continued)

\begin{tabular}{|c|c|c|c|c|c|c|c|c|c|c|c|c|}
\hline & & $\begin{array}{c}\text { Return } \\
(1)\end{array}$ & $\begin{array}{l}\text { SD } \\
(2)\end{array}$ & $\begin{array}{l}\text { Skewness } \\
\text { (3) }\end{array}$ & $\begin{array}{l}\text { Kurtosis } \\
\text { (4) }\end{array}$ & $\begin{array}{c}\mathrm{VaR} \\
(5)\end{array}$ & $\begin{array}{c}\mathrm{CVaR} \\
(6)\end{array}$ & $\begin{array}{c}\text { AHI } \\
(7)\end{array}$ & $\begin{array}{l}\text { IR } \\
(8)\end{array}$ & $\begin{array}{l}\text { SR } \\
(9)\end{array}$ & $\begin{array}{c}\mu / \operatorname{VaR} \\
(10)\end{array}$ & $\begin{array}{l}\mu / C V a R \\
\text { (11) }\end{array}$ \\
\hline \multicolumn{13}{|c|}{$\begin{array}{l}\text { Panel F. The MCVaR-BL portfolio } \\
\text { The MCVaR-BL portfolio (99\% Confidence Level) }\end{array}$} \\
\hline \multirow{3}{*}{ MCVaR-BL N } & DCC & 0.007 & 0.039 & -0.460 & 4.041 & 0.098 & 0.116 & 0.154 & 0.119 & 0.190 & 0.076 & 0.064 \\
\hline & EWMA & 0.007 & 0.038 & -0.237 & 4.784 & 0.100 & 0.118 & 0.098 & 0.088 & 0.173 & 0.066 & 0.056 \\
\hline & RW & 0.007 & 0.038 & -0.271 & 4.399 & 0.100 & 0.105 & 0.103 & 0.100 & 0.170 & 0.065 & 0.062 \\
\hline \multirow{3}{*}{ MCVaR-BL t } & DCC & 0.008 & 0.039 & -0.436 & 4.063 & 0.098 & 0.116 & 0.164 & 0.143 & 0.207 & 0.083 & 0.069 \\
\hline & EWMA & 0.007 & 0.038 & -0.232 & 4.766 & 0.100 & 0.117 & 0.098 & 0.088 & 0.173 & 0.066 & 0.056 \\
\hline & RW & 0.007 & 0.038 & -0.272 & 4.390 & 0.100 & 0.105 & 0.103 & 0.098 & 0.170 & 0.065 & 0.062 \\
\hline \multicolumn{13}{|c|}{ Panel F2. The MCVaR-BL portfolio (95\% Confidence Level) } \\
\hline \multirow{3}{*}{ MCVaR-BL N } & DCC & 0.008 & 0.039 & -0.451 & 4.127 & 0.098 & 0.116 & 0.158 & 0.131 & 0.196 & 0.079 & 0.066 \\
\hline & EWMA & 0.007 & 0.038 & -0.233 & 4.798 & 0.100 & 0.118 & 0.098 & 0.088 & 0.173 & 0.066 & 0.056 \\
\hline & RW & 0.007 & 0.038 & -0.271 & 4.398 & 0.100 & 0.105 & 0.103 & 0.100 & 0.170 & 0.065 & 0.062 \\
\hline \multirow{3}{*}{ MCVaR-BL t } & DCC & 0.008 & 0.039 & -0.452 & 4.124 & 0.098 & 0.116 & 0.158 & 0.130 & 0.196 & 0.079 & 0.066 \\
\hline & EWMA & 0.007 & 0.038 & -0.237 & 4.782 & 0.100 & 0.118 & 0.098 & 0.088 & 0.173 & 0.066 & 0.056 \\
\hline & RW & 0.007 & 0.038 & -0.274 & 4.390 & 0.100 & 0.105 & 0.103 & 0.099 & 0.170 & 0.065 & 0.062 \\
\hline \multicolumn{13}{|c|}{ Panel F3. The MCVaR-BL portfolio (90\% Confidence Level) } \\
\hline \multirow{3}{*}{ MCVaR-BL N } & DCC & 0.008 & 0.039 & -0.444 & 4.126 & 0.098 & 0.116 & 0.157 & 0.131 & 0.196 & 0.079 & 0.066 \\
\hline & EWMA & 0.007 & 0.038 & -0.236 & 4.805 & 0.100 & 0.118 & 0.098 & 0.088 & 0.173 & 0.066 & 0.056 \\
\hline & RW & 0.007 & 0.038 & -0.272 & 4.399 & 0.100 & 0.105 & 0.103 & 0.100 & 0.170 & 0.065 & 0.062 \\
\hline \multirow{3}{*}{ MCVaR-BL t } & DCC & 0.008 & 0.039 & -0.452 & 4.127 & 0.098 & 0.116 & 0.159 & 0.131 & 0.197 & 0.079 & 0.066 \\
\hline & EWMA & 0.007 & 0.038 & -0.236 & 4.785 & 0.100 & 0.118 & 0.098 & 0.088 & 0.173 & 0.066 & 0.056 \\
\hline & RW & 0.007 & 0.038 & -0.271 & 4.399 & 0.100 & 0.105 & 0.103 & 0.100 & 0.170 & 0.065 & 0.062 \\
\hline
\end{tabular}




\section{Table 4 Out-of-sample Risk-Adjusted Unconstrained BL Portfolios Performance}

Table 4 reports realized risk-adjusted unconstrained BL portfolio performance in the period from August 2003 to August 2015 . Return is the average realized excess return, Sharpe Ratio (SR) is the average excess realized return divided by the standard deviation (SD). Average Herfindahl index (AHI) is the average value of the time-varying Herfindahl index. Information Ratio (IR) is the average active return divided by the standard deviation of active return. Both $\mathrm{VaR}$ and $\mathrm{CVaR}$ are measured on the empirical distribution. $\mu / V a R$ and $\mu / C V a R$ evaluate the excess return per unit of tail risk. In the construction of the portfolio, both VaR and CVaR are estimated by the parametric method with the assumption of a normal distribution (' $N$ ') and t-distribution (' $t$ ') at confidence levels of $99 \%, 95 \%$ and $90 \%$. The implied variance-adjusted BL portfolio is constructed by reverse optimization of the utility function. The variance-adjusted SR-BL portfolio is constructed by maximizing the SR. The $\pi$-VaR portfolio is constructed by maximizing the VaR-adjusted return to SD ratio. The $\pi$-CVaR portfolio is constructed by maximizing the CVaR-adjusted return to SD ratio.

\begin{tabular}{|c|c|c|c|c|c|c|c|c|c|c|c|}
\hline & $\begin{array}{c}\text { Return } \\
(1) \\
\end{array}$ & $\begin{array}{l}\text { SD } \\
(2) \\
\end{array}$ & $\begin{array}{c}\text { Skewness } \\
(3) \\
\end{array}$ & $\begin{array}{c}\text { Kurtosis } \\
(4)\end{array}$ & $\begin{array}{c}\text { VaR } \\
(5) \\
\end{array}$ & $\begin{array}{c}\text { CVaR } \\
(6) \\
\end{array}$ & $\begin{array}{c}\text { AHI } \\
(7) \\
\end{array}$ & $\begin{array}{l}\text { IR } \\
(8) \\
\end{array}$ & $\begin{array}{l}\text { SR } \\
(9) \\
\end{array}$ & $\begin{array}{c}/ \mathrm{VaR} \\
(10) \\
\end{array}$ & $\begin{array}{c}\mu / C V a R \\
(11)\end{array}$ \\
\hline \multicolumn{12}{|c|}{ Panel A. The implied variance-adjusted BL portfolio } \\
\hline Implied variance-adjusted BL & 0.009 & 0.043 & -0.363 & 4.278 & 0.100 & 0.136 & 0.307 & 0.094 & 0.206 & 0.088 & 0.065 \\
\hline Variance-adjusted SR-BL & 0.007 & 0.050 & -2.015 & 15.205 & 0.143 & 0.264 & 0.324 & 0.036 & 0.136 & 0.047 & 0.025 \\
\hline \multicolumn{12}{|c|}{ Panel C. The $\pi$-VaR portfolio } \\
\hline$\pi-\mathrm{VaR} \mathrm{t}$ & 0.008 & 0.045 & -0.658 & 6.454 & 0.128 & 0.178 & 0.381 & 0.080 & 0.180 & 0.063 & 0.045 \\
\hline \multicolumn{12}{|c|}{ Panel C2. The $\pi$-VaR portfolio (95\% Confidence Level) } \\
\hline$\pi-\operatorname{VaR} \mathrm{N}$ & 0.008 & 0.077 & -0.732 & 7.512 & 0.297 & 0.313 & 0.546 & 0.037 & 0.105 & 0.027 & 0.026 \\
\hline$\pi-V a R \mathrm{t}$ & 0.009 & 0.051 & -0.623 & 7.095 & 0.132 & 0.207 & 0.429 & 0.098 & 0.179 & 0.069 & 0.044 \\
\hline \multicolumn{12}{|c|}{ Panel C3. The $\pi$-VaR portfolio (90\% Confidence Level) } \\
\hline
\end{tabular}

Table 4 (continued) 
Table 4 (continued)

\begin{tabular}{|c|c|c|c|c|c|c|c|c|c|c|c|}
\hline & $\begin{array}{c}\text { Return } \\
\text { (1) }\end{array}$ & $\begin{array}{l}\mathrm{SD} \\
(2)\end{array}$ & $\begin{array}{c}\text { Skewness } \\
\text { (3) }\end{array}$ & $\begin{array}{c}\text { Kurtosis } \\
(4)\end{array}$ & $\begin{array}{c}\mathrm{VaR} \\
(5)\end{array}$ & $\begin{array}{c}\text { CVaR } \\
(6)\end{array}$ & $\begin{array}{c}\text { AHI } \\
(7)\end{array}$ & $\begin{array}{l}\text { IR } \\
(8) \\
\end{array}$ & $\begin{array}{l}\text { SR } \\
(9) \\
\end{array}$ & $\begin{array}{c}\mu / \mathrm{VaR} \\
(10)\end{array}$ & $\begin{array}{c}\mu / C V a R \\
(11)\end{array}$ \\
\hline \multicolumn{12}{|c|}{ Panel D. The $\pi$-CVaR portfolio } \\
\hline \multicolumn{12}{|c|}{ Panel D1. The $\pi$-CVaR portfolio (99\% Confidence Level) } \\
\hline$\pi-\mathrm{CVaR} N$ & 0.010 & 0.054 & -0.252 & 5.985 & 0.130 & 0.192 & 0.425 & 0.116 & 0.183 & 0.076 & 0.051 \\
\hline$\pi-\mathrm{CVaR} t$ & 0.009 & 0.048 & -0.372 & 5.491 & 0.127 & 0.170 & 0.382 & 0.104 & 0.187 & 0.070 & 0.052 \\
\hline \multicolumn{12}{|c|}{ Panel D2. The $\pi$-CVaR portfolio (95\% Confidence Level) } \\
\hline$\pi-\mathrm{CVaR} N$ & 0.013 & 0.076 & 0.478 & 7.183 & 0.220 & 0.237 & 0.541 & 0.138 & 0.174 & 0.060 & 0.056 \\
\hline$\pi-\mathrm{CVaR} \mathrm{t}$ & 0.009 & 0.051 & -0.337 & 5.812 & 0.129 & 0.184 & 0.407 & 0.110 & 0.185 & 0.073 & 0.051 \\
\hline \multicolumn{12}{|c|}{ Panel D3. The $\pi$-CVaR portfolio (90\% Confidence Level) } \\
\hline$\pi-\mathrm{CVaR} N$ & 0.008 & 0.048 & -1.226 & 10.300 & 0.135 & 0.225 & 0.431 & 0.066 & 0.165 & 0.059 & 0.035 \\
\hline$\pi-\mathrm{CVaR} t$ & 0.010 & 0.056 & -0.182 & 6.060 & 0.138 & 0.198 & 0.436 & 0.120 & 0.182 & 0.074 & 0.051 \\
\hline
\end{tabular}


Table 5 Out-of-sample Dynamic BL Portfolios Performance Ranking

Table 5 summarises out-of-sample performance evaluation ratios and report the rankings of the benchmark portfolio, 1/N portfolio, the the DCCbased BL portfolios in the period from August 2003 to August 2015. Sharpe Ratio (SR) is the average excess realized return divided by the standard deviation (SD). Average Herfindahl index (AHI) is the average value of the time-varying Herfindahl index. Information Ratio (IR) is the average active return divided by the standard deviation of active return. Both $\mathrm{VaR}$ and $\mathrm{CVaR}$ are measured on the empirical distribution. $\mu / \operatorname{VaR}$ and $\mu / C V a R$ evaluate the excess return per unit of tail risk. The implied BL portfolio is constructed by reverse optimization of the utility function. The SR-BL portfolio is constructed by maximizing the SR. The MVaR-BL portfolio is constructed by maximizing the rewardto-VaR ratio. The MCVaR-BL portfolio is constructed by maximizing the reward-to-CVaR ratio. The implied variance-adjusted BL portfolio is constructed by reverse optimization of the utility function. The variance-adjusted SR-BL portfolio is constructed by maximizing the Varianceadjusted return to SD ratio. The $\pi-V a R$ portfolio is constructed by maximizing the VaR-adjusted return to SD ratio. The $\pi-C V a R$ portfolio is constructed by maximizing the CVaR-adjusted return to SD ratio. Both VaR and CVaR are estimated by the parametric method with the assumption of a normal distribution ('N') or t-distribution (' $\mathrm{t}$ ') at a confidence level of $99 \%$.

\begin{tabular}{|c|c|c|c|c|c|c|c|c|c|c|}
\hline & SR & Ranking & $\mu / \mathrm{VaR}$ & Ranking & $\mu / C V a R$ & Ranking & AHI & Ranking & IR & Ranking \\
\hline Benchmark & 0.135 & 12 & 0.049 & 11 & 0.036 & 11 & 0.075 & 2 & $\mathrm{NaN}$ & $\mathrm{NaN}$ \\
\hline $1 / \mathrm{N}$ & 0.118 & 14 & 0.036 & 13 & 0.030 & 12 & $\mathbf{0 . 0 3 3}$ & 1 & -0.070 & 13 \\
\hline Implied BL & 0.199 & 4 & 0.078 & 5 & 0.074 & 1 & 0.094 & 5 & 0.157 & 1 \\
\hline MVaR 0.99 N & 0.196 & 5 & 0.079 & 4 & 0.066 & 4 & 0.079 & 3 & 0.130 & 4 \\
\hline MVaR 0.99 t & 0.200 & 3 & 0.080 & 3 & 0.067 & 3 & 0.080 & 4 & 0.137 & 3 \\
\hline Implied variance-adjusted $B L$ & 0.206 & 2 & 0.088 & 1 & 0.065 & 5 & 0.307 & 9 & 0.094 & 8 \\
\hline Variance-adjusted SR-BL & 0.136 & 11 & 0.047 & 12 & 0.025 & 13 & 0.324 & 10 & 0.036 & 12 \\
\hline VaR-adjusted SR-BL $0.99 \mathrm{~N}$ & 0.126 & 13 & 0.025 & 14 & 0.024 & 14 & 0.541 & 14 & 0.046 & 11 \\
\hline VaR-adjusted SR-BL $0.99 \mathrm{t}$ & 0.180 & 9 & 0.063 & 9 & 0.045 & 9 & 0.381 & 11 & 0.080 & 9 \\
\hline CVaR-adjusted SR-BL $0.99 \mathrm{~N}$ & 0.183 & 8 & 0.076 & 7 & 0.051 & 8 & 0.425 & 13 & 0.116 & 6 \\
\hline CVaR-adjusted SR-BL $0.99 \mathrm{t}$ & 0.187 & 7 & 0.070 & 8 & 0.052 & 7 & 0.382 & 12 & 0.104 & 7 \\
\hline
\end{tabular}


Table 6 Out-of-sample of the Dynamic BL Portfolios Using Different Window Lengths and Different Data Frequencies

Table 6 summarises out-of-sample performance evaluation ratios for the benchmark portfolio, 1/N portfolio, and selected DCC-based BL portfolios in the period from August 2003 to August 2015, using an estimation window of 36 months, 60 months and 110 months. Sharpe Ratio (SR) is the average excess realized return divided by the standard deviation (SD). Information Ratio (IR) is the average active return divided by the standard deviation of active return. Both $\mathrm{VaR}$ and $\mathrm{CVaR}$ are measured using the empirical distribution. $\mu / V a R$ and $\mu / C V a R$ evaluate the excess return per unit of tail risk. The implied BL portfolio is constructed by reverse optimization of the utility function. The MVaR-BL portfolio is constructed by maximizing the reward-to-VaR ratio. The MCVaR-BL portfolio is constructed by maximizing the reward-to-CVaR ratio. The implied variance-adjusted BL portfolio is constructed by reverse optimization of the utility function. The $\pi-V a R$ portfolio is constructed by maximizing the VaR-adjusted return to SD ratio. The $\pi$-CVaR portfolio is constructed by maximizing the CVaR-adjusted return to SD ratio. Both $\mathrm{VaR}$ and $\mathrm{CVaR}$ are estimated by the parametric method with the assumption of a t-distribution (' $\mathrm{t}$ ') at a confidence level of 99\%.

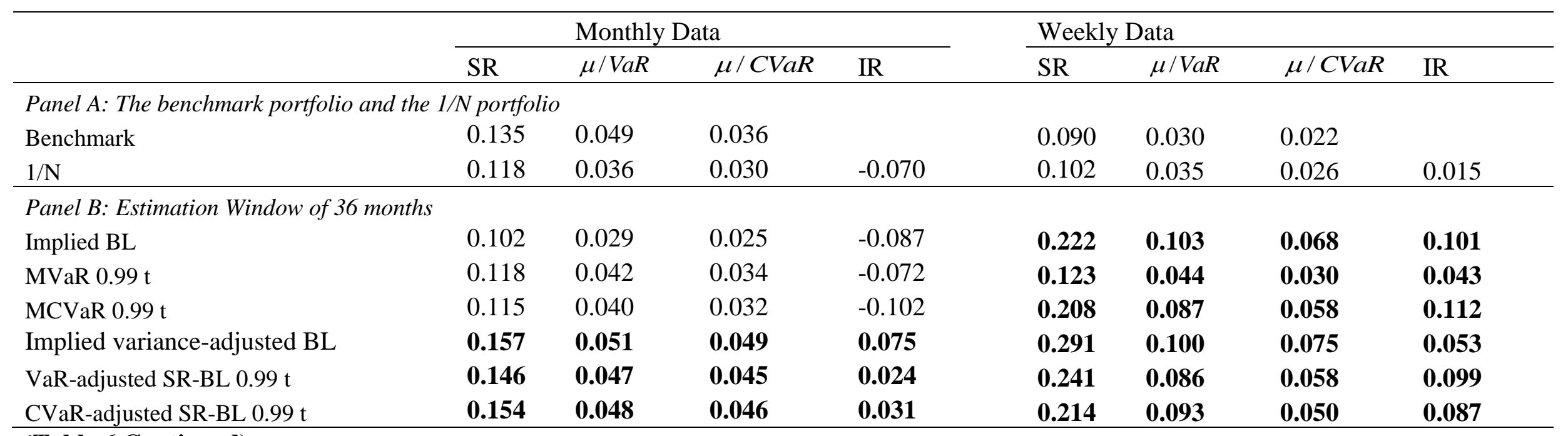

(Table 6 Continued) 
(Table 6 Continued)

\begin{tabular}{|c|c|c|c|c|c|c|c|c|}
\hline & \multicolumn{4}{|c|}{ Monthly Data } & \multicolumn{4}{|c|}{ Weekly Data } \\
\hline & SR & $\mu / V a R$ & $\mu / C V a R$ & IR & SR & $\mu / \operatorname{VaR}$ & $\mu / C V a R$ & IR \\
\hline \multicolumn{9}{|c|}{ Panel C: Estimation Window of 60 months } \\
\hline Implied BL & 0.190 & 0.069 & 0.060 & 0.109 & 0.183 & 0.081 & 0.066 & 0.061 \\
\hline MCVaR $0.99 \mathrm{t}$ & 0.137 & 0.049 & 0.041 & -0.022 & 0.087 & 0.028 & 0.021 & -0.018 \\
\hline Implied variance-adjusted BL & 0.152 & $\mathbf{0 . 0 5 0}$ & 0.047 & 0.049 & 0.169 & 0.070 & 0.041 & -0.006 \\
\hline VaR-adjusted SR-BL $0.99 \mathrm{t}$ & 0.157 & 0.052 & 0.048 & 0.062 & 0.182 & 0.065 & 0.042 & 0.079 \\
\hline \multicolumn{9}{|c|}{ Panel D: Estimation Window of 110 months } \\
\hline Implied BL & 0.199 & 0.078 & 0.074 & 0.157 & 0.178 & 0.077 & 0.054 & 0.065 \\
\hline MVaR $0.99 \mathrm{t}$ & 0.200 & 0.080 & 0.067 & 0.137 & 0.037 & 0.012 & 0.008 & -0.074 \\
\hline MCVaR $0.99 \mathrm{t}$ & 0.207 & $\mathbf{0 . 0 8 3}$ & 0.069 & 0.143 & -0.021 & -0.009 & -0.004 & -0.056 \\
\hline Implied variance-adjusted BL & 0.206 & 0.088 & 0.065 & 0.094 & 0.235 & 0.090 & 0.064 & 0.010 \\
\hline VaR-adjusted SR-BL $0.99 \mathrm{t}$ & 0.180 & 0.063 & 0.045 & $\mathbf{0 . 0 8 0}$ & 0.163 & 0.057 & 0.042 & 0.104 \\
\hline
\end{tabular}


Table 7 Out-of-sample Dynamic BL Portfolios Performance in Sub-periods

Table 7 reports out-of-sample performance of the benchmark portfolio, 1/N portfolio, and the DCC-based BL portfolios in three sub-periods between August 2003 and August 2015. The results are based on weekly data with an estimation window length of 36 months. Return is the average realized excess return, Sharpe Ratio (SR) is the average excess realized return divided by the standard deviation (SD). Average Herfindahl index (AHI) is the average value of the time-varying Herfindahl index. Information Ratio (IR) is the average active return divided by the standard deviation of active return. Both $\mathrm{VaR}$ and $\mathrm{CVaR}$ are measured on the empirical distribution. $\mu / V a R$ and $\mu / C V a R$ evaluate the excess return per unit of tail risk. The implied BL portfolio is constructed by reverse optimization of the utility function. The SR-BL portfolio is constructed by maximizing the SR. The MVaR-BL portfolio is constructed by maximizing the reward-to-VaR ratio. The MCVaR-BL portfolio is constructed by maximizing the reward-to-CVaR ratio. The implied variance-adjusted BL portfolio is constructed by reverse optimization of the utility function. The variance-adjusted SR-BL portfolio is constructed by maximizing the Variance-adjusted return to SD ratio. The $\pi$-VaR portfolio is constructed by maximizing the VaR-adjusted return to SD ratio. The $\pi$-CVaR portfolio is constructed by maximizing the CVaRadjusted return to SD ratio. Both $\mathrm{VaR}$ and $\mathrm{CVaR}$ are estimated by the parametric method with the assumption of a normal distribution ('N') or tdistribution (' $\mathrm{t}$ ') at a confidence level of $99 \%$.

\begin{tabular}{|c|c|c|c|c|c|c|c|c|c|c|c|}
\hline & Return & $\mathrm{SD}$ & Skewness & Kurtosis & $\mathrm{VaR}$ & $\mathrm{CVaR}$ & $\mathrm{AHI}$ & IR & SR & $\mu / V a R$ & $\mu / C V a R$ \\
\hline & $(1)$ & $(2)$ & $(3)$ & (4) & $(5)$ & $(6)$ & $(7)$ & $(8)$ & (9) & $(10)$ & $(11)$ \\
\hline \multicolumn{12}{|c|}{ Panel A. Sub-period 1: August 2003 to October 2007} \\
\hline Benchmark & 0.008 & 0.031 & -0.224 & 0.835 & 0.085 & 0.094 & 0.081 & $\mathrm{NaN}$ & 0.243 & 0.089 & 0.080 \\
\hline $1 / \mathrm{N}$ & 0.011 & 0.034 & -0.183 & 0.825 & 0.086 & 0.105 & 0.033 & 0.125 & 0.313 & 0.125 & 0.102 \\
\hline Implied BL & 0.009 & 0.030 & -0.244 & 0.845 & 0.083 & 0.091 & 0.137 & 0.147 & 0.292 & 0.107 & 0.096 \\
\hline SR-BL & 0.009 & 0.030 & -0.253 & 0.857 & 0.084 & 0.093 & 0.149 & 0.122 & 0.285 & 0.102 & 0.093 \\
\hline mu-VaR $0.99 \mathrm{~N}$ & 0.009 & 0.030 & -0.254 & 0.857 & 0.084 & 0.093 & 0.148 & 0.119 & 0.284 & 0.102 & 0.093 \\
\hline mu-VaR $0.99 \mathrm{t}$ & 0.008 & 0.030 & -0.233 & 0.845 & 0.085 & 0.091 & 0.079 & 0.051 & 0.274 & 0.096 & 0.090 \\
\hline mu-CVaR $0.99 \mathrm{~N}$ & 0.009 & 0.030 & -0.221 & 0.835 & 0.083 & 0.085 & 0.930 & 0.003 & 0.316 & 0.114 & 0.111 \\
\hline mu-CVaR $0.99 \mathrm{t}$ & 0.009 & 0.030 & -0.257 & 0.858 & 0.085 & 0.093 & 0.079 & 0.111 & 0.284 & 0.101 & 0.093 \\
\hline Implied variance-adjusted BL & 0.004 & 0.010 & -0.119 & 1.208 & 0.025 & 0.035 & 0.063 & -0.069 & 0.360 & 0.150 & 0.107 \\
\hline Variance-adjusted SR-BL & 0.015 & 0.039 & -0.048 & 0.924 & 0.097 & 0.107 & 0.353 & 0.118 & 0.380 & 0.152 & 0.138 \\
\hline
\end{tabular}

(Table 7 continued) 
(Table 7 continued)

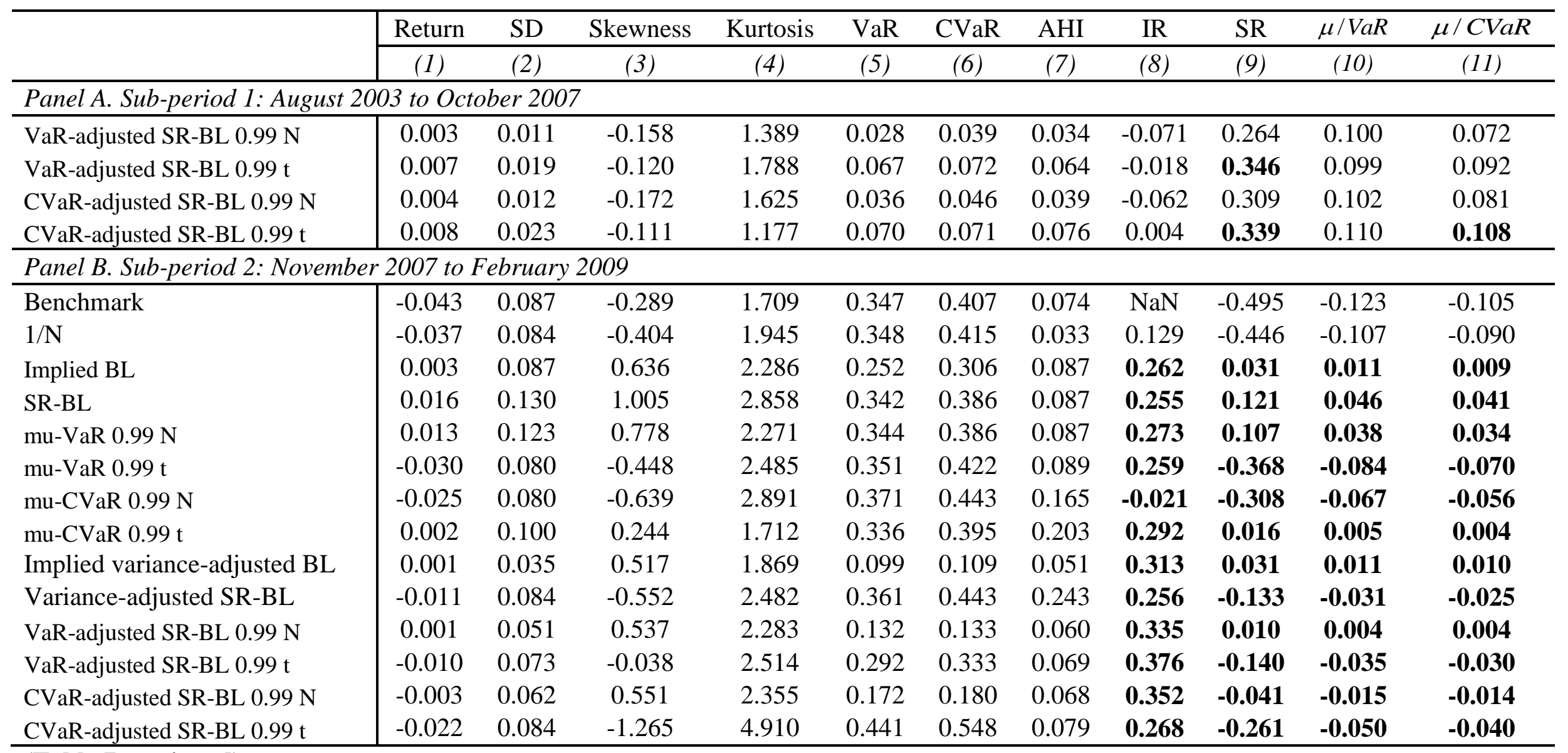

\footnotetext{
(Table 7 continued)
} 
(Table 7 continued)

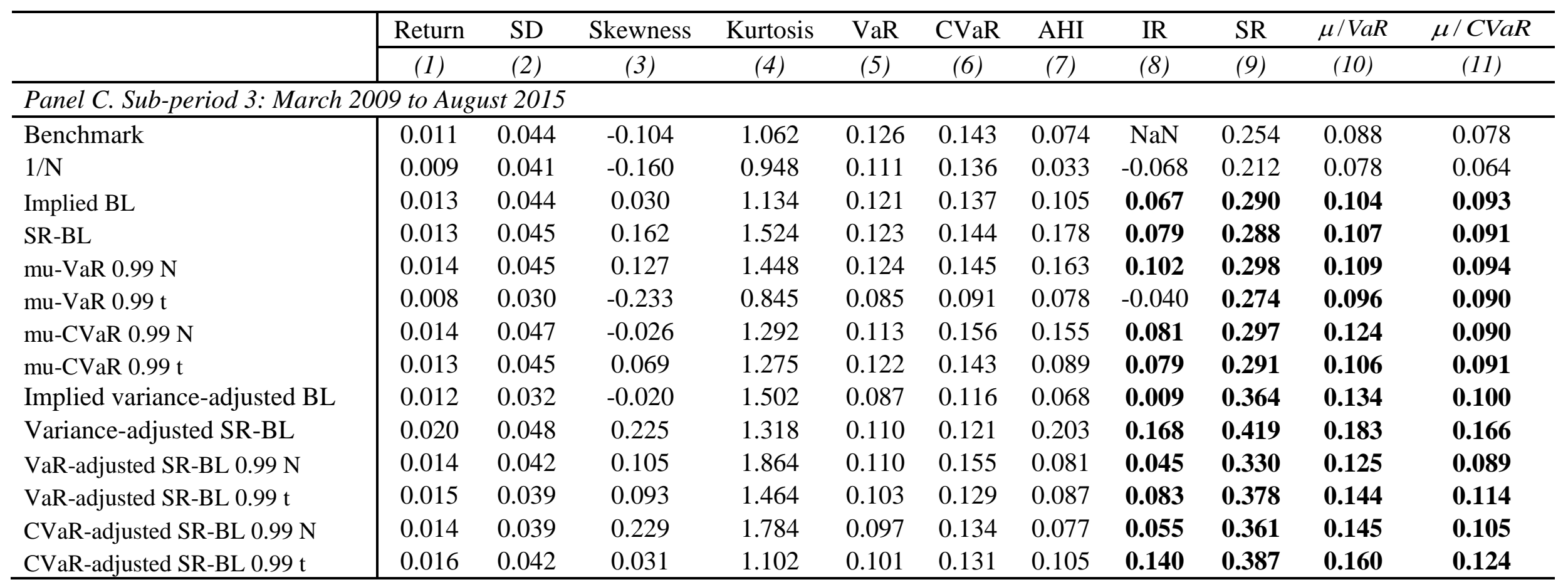

